\title{
A new view on the M 87 jet origin: Turbulent loading leading to large-scale episodic wiggling
}

\author{
S. Britzen ${ }^{1}$, C. Fendt ${ }^{2}$, A. Eckart ${ }^{1,3}$, and V. Karas ${ }^{4}$ \\ ${ }^{1}$ Max-Planck-Institut für Radioastronomie, Auf dem Hügel 69, 53121 Bonn, Germany \\ e-mail: sbritzen@mpifr.de \\ 2 Max-Planck-Institut für Astronomie, Königstuhl, 69117 Heidelberg, Germany \\ 3 I. Physikalisches Institut der Universität zu Köln, Zülpicher Str. 77, 50937 Köln, Germany \\ 4 Astronomical Institute, Academy of Sciences, Boční II 1401, 14131 Prague, Czech Republic
}

Received 4 August 2016 / Accepted 19 January 2017

\begin{abstract}
Context. The nearby, giant radio galaxy M 87 hosts a supermassive black hole $(\mathrm{BH})$ and is well-known for a bright jet dominating the spectrum over ten orders of magnitude in frequency. Due to its proximity, jet prominence, and the large $\mathrm{BH}$ mass, M 87 is the best laboratory for investigating the formation, acceleration, and collimation of relativistic jets. Many kinematic studies have been performed to determine the proper motions in the jet. Despite M 87 providing all proofs of being an active BH, the apparent jet speed remained puzzling, because proper motion measurements between 15 and $43 \mathrm{GHz}$ for the same region of 1-10 mas core distance provided largely discrepant results. This source is a prime object to be studied in exquisite detail with the upcoming Event Horizon Telescope (EHT) observations because it promises to allow a direct view on the jet launching process itself.

Aims. We aim to decipher some of the kinematic puzzles in the pc-scale jet with the analysis we present here.

Methods. We re-modeled and re-analyzed 31 VLBA observations at $15 \mathrm{GHz}$ obtained within the MOJAVE program. The data span a time range between Jul. 1995 and May 2011. We performed a detailed investigation of the pc-scale jet kinematics on different timescales, the shortest periods between the observations beeing 10 and 80 days, and in different jet modes, making use of VLBA observations. In addition, we studied the jet ridge line behavior as a function of time. Special care was taken to analyze the region close to the $15 \mathrm{GHz}$ core, and the dynamics and distribution of newly emerging jet features in the jet.

Results. We find an indication for apparent superluminal motion in the jet. Moreover, we present evidence for acceleration between 0.5 and 10 mas of core separation. The data suggest that the central part of M 87 at $15 \mathrm{GHz}$ seems to be rotating. Jet components and counter-jet components are ejected in different directions under varying angles, explaining the impression of a broad opening angle. In this paper we present evidence for two different operating modes of the jet of M 87. The jet switches between two phases: i) the jet ridge line is at least double or the jet axis is displaced vertically, and ii) an unperturbed phase where the jet ridge line remains almost straight but is smoothly curved and the jet components are aligned along a classical jet axis. The mode change occurs every couple of years. Between the two operating modes, a transition phase is visible.

Conclusions. The M 87 jet visible at $15 \mathrm{GHz}$ probes a different physical zone compared to the standard blazar-zone we tend to see in AGN jets. The most likely scenario explaining the observed phenomena is a turbulent mass loading into the jet, most probably due to local, fast reconnection processes driven by turbulence of a tangled magnetic field, which is either generated in the accretion disk or the disk corona. In addition, on large scales, a global magnetic structure is required to channel the turbulent flow into what evolves into a large-scale jet. Large-scale jet instabilities may explain the curved pattern of the observed jet flow.
\end{abstract}

Key words. techniques: interferometric - galaxies: active - galaxies: jets

\section{Introduction: The prominent jet of $M 87$ and its apparent velocities}

M 87 is the central elliptical galaxy in the Virgo cluster and at a distance of $16.7 \mathrm{Mpc}$ the second closest active black hole to our galaxy. The bright jet and counter-jet have been studied intensively in all wavelength regimes, radio to $\mathrm{TeV}$. The most detailed information for this showcase jet has been obtained in the radio regime with an increase in observing frequencies and resolution in recent years (e.g., Biretta \& Junor 1995; Walker et al. 2008; Hada et al. 2016). The jet is also visible in the optical (HST, e.g., Ford et al. 1994) and in the X-ray (e.g., Wilson et al. 2002) regime. Wagner et al. (2009) pinpointed the TeV gamma-ray emission region in $\mathrm{M} 87$ using $\mathrm{TeV}$ and very long baseline array (VLBA) monitoring at $43 \mathrm{GHz}$. M 87 was the first radio galaxy to be detected in the $\mathrm{TeV}$ regime. The temporal coincidence of the $\mathrm{TeV}$ and radio flares indicate an origin of the $\mathrm{TeV}$ radiation within a few tens of Schwarzschild radii of the radio core. M 87 will be studied with highest achievable angular resolution with the upcoming Event Horizon Telescope (EHT) observations because it promises a direct view on the jet launching process itself (e.g., Broderick et al. 2011; Lu et al. 2014; Fish et al. 2016).

Many kinematic studies have been performed to determine the proper motions in the jet. Observations of the pc-scale jet of M 87 by Craig Walker et al. (2008) reveal in a super-resolved (in north-south direction) image a wide jet base.

Although the details seem disturbed, the structure is symmetric between the jet and counter-jet. The counter-jet side is shorter, as expected from beaming arguments. Several very long baseline interferometry (VLBI) observations show evidence 


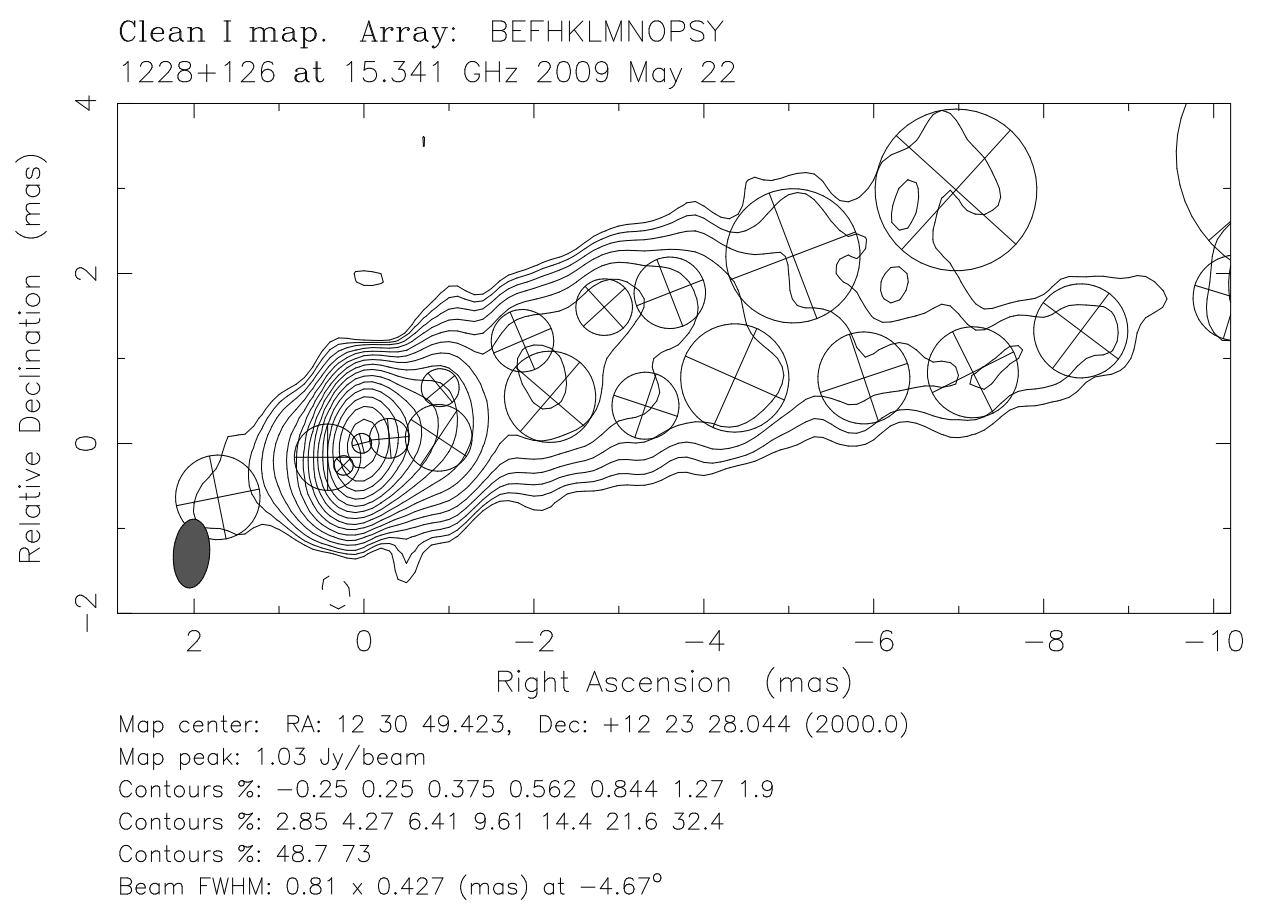

Fig. 1. Highest quality map showing the typical structure of the pc-scale jet at $15 \mathrm{GHz}$.

for slow motions. Biretta \& Junor (1995) and Junor \& Biretta (1995) find apparent speeds slower than $0.1 c$ based on VLBA observations. Dodson et al. (2006) find no evidence for motions based on VLBI space observatory programme (VSOP) observations. Reid et al. (1989) determine a value of $0.28 c$ based on VLBI observations at $1.6 \mathrm{GHz}$, and Ly et al. (2007) find values between 0.25 and $0.4 c$ based on VLBA observations at $43 \mathrm{GHz}$. Proper motion measurements between 15 and $43 \mathrm{GHz}$ for the same region of 1-10 mas core distance provided largely discrepant results (for a discussion please see Asada et al. 2014). Superluminal motions up to $2 c$ were detected by VLBA observations at $43 \mathrm{GHz}$ (Walker et al. 2008), whereas VLBA and VLBI observations at other frequencies, including $15 \mathrm{GHz}$, detected no or only slow subluminal motions (e.g., Kovalev et al. 2007, based on MOJAVE monitoring data with sampling interval $5 \pm 3$ months).

Mertens et al. (2015) study the two-dimensional structure and kinematics of M 87 by applying the Wavelet-based Image Segmentation and Evaluation (WISE) method to eleven images obtained from VLBA observations at $43 \mathrm{GHz}$. They find a linear acceleration regime up to $z_{\mathrm{obs}} \sim 2$ mas. The acceleration is reduced at larger scales, consistent with saturation of Poynting flux conversion according to the authors. A stacked cross-correlation analysis of the images reveals a pronounced stratification of the flow. According to them, the flow consists of a slow, mildly relativistic layer (moving at $\beta \sim 0.5 c$ ), associated either with instability pattern speed or an outer wind, and a fast, accelerating stream line (with $\beta \sim 0.92$, corresponding to a bulk Lorentz factor $\gamma \sim 2.5$ ). They find a systematic difference of the apparent speeds in the northern and southern limbs of the jet.

On larger scales, the kinematic situation in the M 87 jet is different. Biretta et al. (1995) find typical values of $0.5 c$, but also values up to $2.5 c$, in VLA observations. Optical observations (HST, Biretta et al. 1999) yield values for the knot at $0.9^{\prime \prime}$ (70 pc projected) of about 5-6 c. Cheung et al. (2007) and Giroletti et al. (2012) find speeds of 2.5-4.5 $c$ based on VLBA observations at $20 \mathrm{~cm}$ downstream to the HST-1 component.

\section{Observations and data analysis}

The MOJAVE survey program obtains exquisite VLBA data at $15 \mathrm{GHz}$ and makes them available for the astronomical community. This service allows detailed studies of individual active galactic nucleus (AGN) jets as well as statistical analysis of a large-enough sample for general AGN properties. The typical time interval of VLBA observations within the MOJAVE program is excellently suited to study the apparent speeds in AGN in general. However, the data sampling rate for the close-by M 87 is insufficient to adequately monitor jet component and counterjet component motion. From inspecting two epochs separated by 10 days, we estimate that a sampling rate of every 5 days may be adequate to determine the kinematics safely.

We re-modeled and re-analyzed 31 VLBA observations (15 GHz, MOJAVE) performed between Jul. 1995 and May 2011. Gaussian circular components were fit to the data to obtain the optimum set of parameters within the difmap-modelfit program (Shepherd 1997). Several identification schemes were tested to most reliably trace the components through the epochs. Uncertainties of the modelfit component parameters were determined by comparing the parameters derived for models with different numbers of components for the same epoch. All maps with modelfit components superimposed are shown in Figs. A.1-A.8. Throughout the paper we adopt the following parameters: $D=16.7 \mathrm{Mpc}, M_{\mathrm{BH}}=6 \times 10^{9} M_{\odot}$ for M 87, corresponding to 1 mas $=0.08 \mathrm{pc}=140 R_{\mathrm{S}}$.

\section{The jet: Episodic oscillations and unperturbed phases}

\subsection{Jet axis displacements \& a swirling jet origin}

The detailed structure of the M 87 jet is shown in Fig. 1 for the epoch of highest quality observations. Our analysis confirms the complexity of this jet on pc-scales (Walker et al. 2008). The jet splits into two regimes parallel to an imaginary axis, often described as limb brightening (e.g., Reid et al. 1989), in this epoch. 


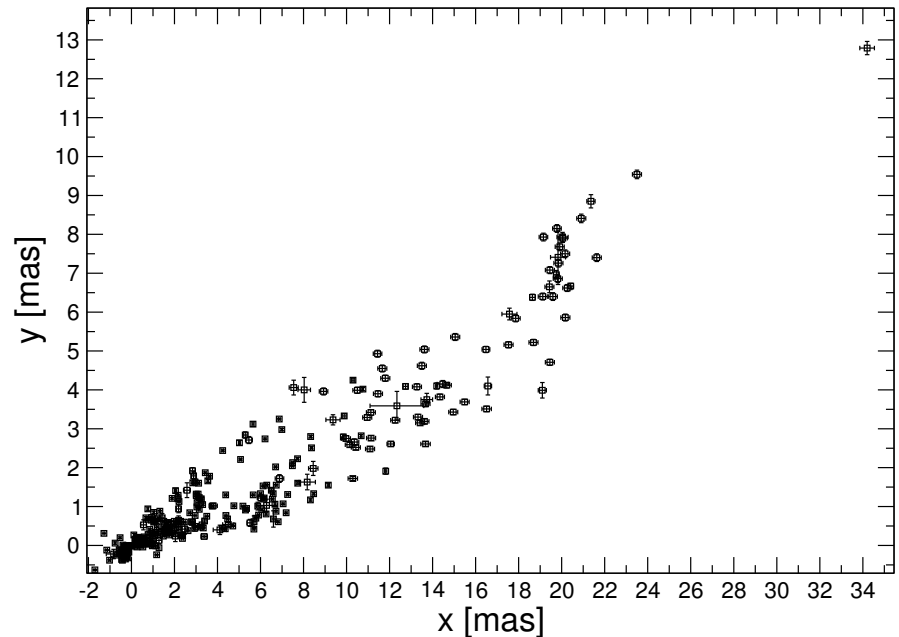

(a)

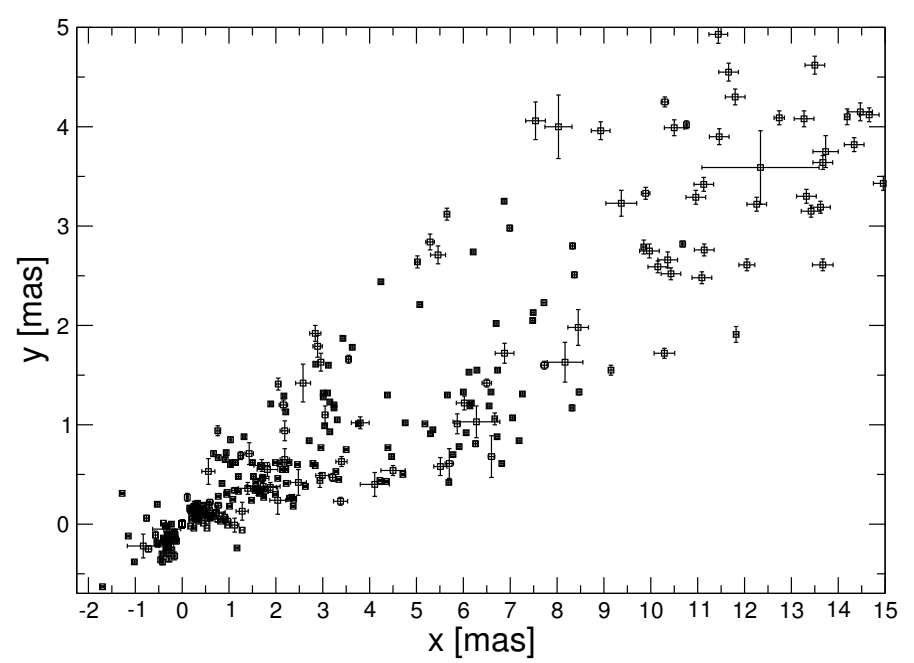

(b)

Fig. 2. Plot a) all components found in the model-fitting process in $x$ versus $y$. Plot b) a zoom into the inner 15 mas in $x$.

This limb-brightened jet is also confirmed at extreme resolution and sensitivity achieved by the Global Millimeter-Very Long Baseline Interferometry Array (GMVA) at 43 and $86 \mathrm{GHz}$ (Kim et al. 2016). In Fig. 2 we show all jet components found in the model-fitting process in all epochs in $x$ versus $y$ (with error bars, upper plot). The lower plot of Fig. 2 shows a zoom-in into the inner 15 mas distance from the core. The limb-brightened structure is not so clearly visible any more. Instead we find transverse structure (indicated in the sketch in Fig. 5).

The jet of M 87 appears in two different modes. At about 1 mas core separation the jet in one of the modes opens up into a broad jet that consists of two strings (limbs). Another mode exists in which the jet remains straight with only one standard jet ridge line. In Fig. 3 we examine the jet ridge line behavior for all epochs. About every two years the jet appears to be in a "straight mode" and is then displaced again. The individual observations deviate in data quality, such as the number of data points, and due to insufficient time sampling. Therefore we might miss a straight or broad phase. Nevertheless, the mode changes we detect seem to be a robust and repetitive effect. The effect does not seem to be influenced by the number of data points (visibilities) nor by the angular resolution (beam size) of the individual epochs. This phenomenon seems also to be independent of the flux-density of the core. The last figure of Fig. 3 shows all straight epochs superimposed. Obviously, not even the straight phases result in the same jet ridge line. After roughly thirteen years the jet ridge line appears to have a similar shape again, such that epochs from 1996 and 2010 coincide up to about 12 mas.

In Fig. 4 we investigate the two-mode phenomenon further. All figures show the same data but out to different core separations. We zoom in from core separations up to 34 mas (top) and down to 8 mas (bottom). It is clearly visible how the straight phases are displaced from the two-string (or broad jet) phases. Figure 6 shows the transition between the two modes in more detail. From a two ridge-line mode (9 Aug. 2004), the jet narrows (but still reveals two ridge lines in 2 Dec. 2004) and is straight in 21 Apr. 2005 in only one ridge-line. In Fig. 7 we show the jet ridge line behavior after a straight (one ridge-line) phase (11 Feb. 2010). On 8 Apr. 2010 the jet broadens up until 21 May 2011, at which point the two ridge-line jet structure is visible again.

Figure 8 shows the time evolution of the $x$ and $y$ coordinates of the component closest to the core, which both indicate a periodic behavior. Based on the minima of the $y$-coordinate (21 May 1999, 29 Dec. 2001, 21 Apr. 2005, 5 Feb. 2007, 5 Jul. 2009) we derive a timescale of $2.53 \pm 0.54 \mathrm{yr}^{-1}$. This yields a speed of about 0.16 mas yr for a potential rotation of the closest jet component to the core, based on an amplitude estimated of 0.2 mas. Figure 9 displays the $(x, y)$ positions of those jet and counter-jet components which are closest to the core. Features closest to the core in every epoch are emitted in different directions into the jet. This explains the broad opening angle of the jet.

\subsection{Apparent superluminal motion and acceleration on timescales of days}

A proper identification of jet components in M 87 requires more frequent observations. We note also that the M 87 jet reveals significantly different kinematics compared to most other AGN. Table 1 lists the projected proper motions and apparent speeds derived via several different approaches.

We investigated in detail those epochs that are separated by 10 and 80 days. We determined proper motions in two ways, from the radial motion and from the motion in $x$ - and $y$-directions. In both closely-spaced epochs we find an indication for apparent superluminal motion and of acceleration along the jet. The apparent velocities of the short-term apparent motion and of the apparent motion in consecutive epochs of a straight jet-phase were calculated by extrapolating the motion derived based on two epochs to a year.

In addition, we determined the apparent motions for those phases during which the jet appears as straight and during which we could identify the components across two epochs. In all three cases we find a strong indication for an acceleration along the jet.

Despite the fast motion we find on short timescales, the longterm data reveal slower apparent motions. In Fig. 10 we mark features traced over longer time spans (J6, J10, J15, J20). Their proper motions, which were determined via linear regression, and their apparent speeds are listed in Table 1.

\subsection{Geometrical considerations}

The viewing angle of M87 is a puzzling question, as is the proper motion (see e.g., Hada et al. 2016). Owen et al. (1989) find a value $\geq 45^{\circ}$ derived from the patterns of helically-wrapped filaments seen in the kpc-scale VLA jet. Based on the proper motion and brightness ratio (VLBA, $43 \mathrm{GHz}$ ), Ly et al. (2007) suggest a value of $30^{\circ}-45^{\circ}$. 

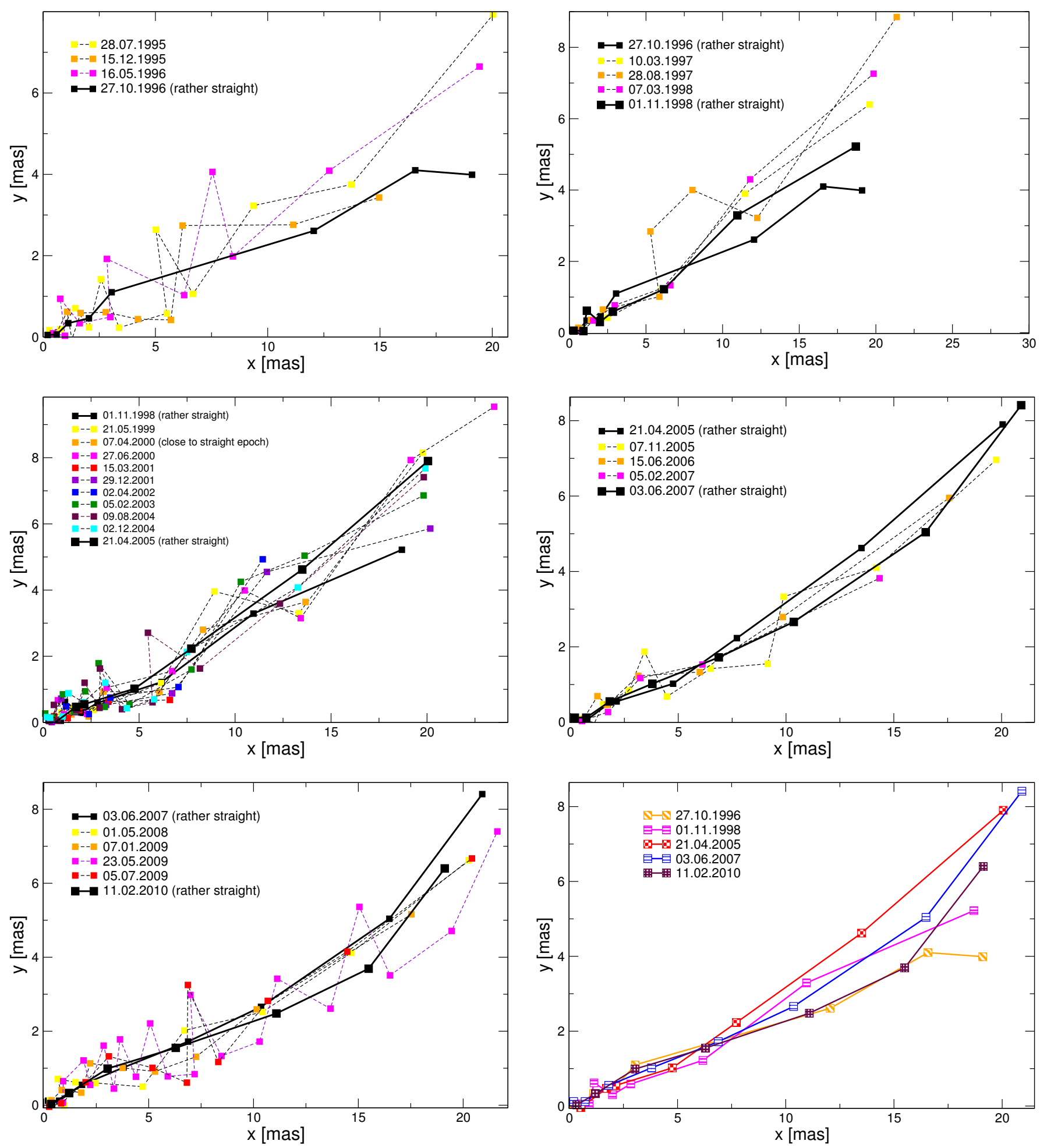

Fig. 3. Jet-ridge-line resulting from connecting all jet features in one epoch per line. Colors mark the broad jet phases, black symbols mark straight modes. The bottom right figure shows all identified straight phases.

Biretta et al. (1999) find superluminal motion of up to $6 c$ in optical observations of HST-1 and derive a value of $<19^{\circ}$. Perlman et al. (2011) suggest an inclination of $11^{\circ}-19^{\circ}$ based on optical polarization properties of HST-1 located at $\sim 0.8^{\prime \prime}$ from the nucleus. Hada et al. (2016) derive values of $29^{\circ}-45^{\circ}$ at the jet base ( $86 \mathrm{GHz}$ VLBA+GBT) based on the apparent motions in jet and counter-jet. They claim that the jet limb-brightening may not be simply explained by a transverse velocity gradient, if the inclination is $10^{\circ}-20^{\circ}$. They also discuss the possibility that the apparent discrepancy of inclination between the inner jet and HST-1 is due to a change in viewing angle that is not constant along the jet.

It is essential to derive the geometry in the launching area from our data. An estimate on the jet inclination can be derived from the beaming equations for relativistic jets, by taking the radial proper motion of the inner knots of jet and counter-jet into consideration and neglecting a tangential motion that is probably caused by rotation or precession. 


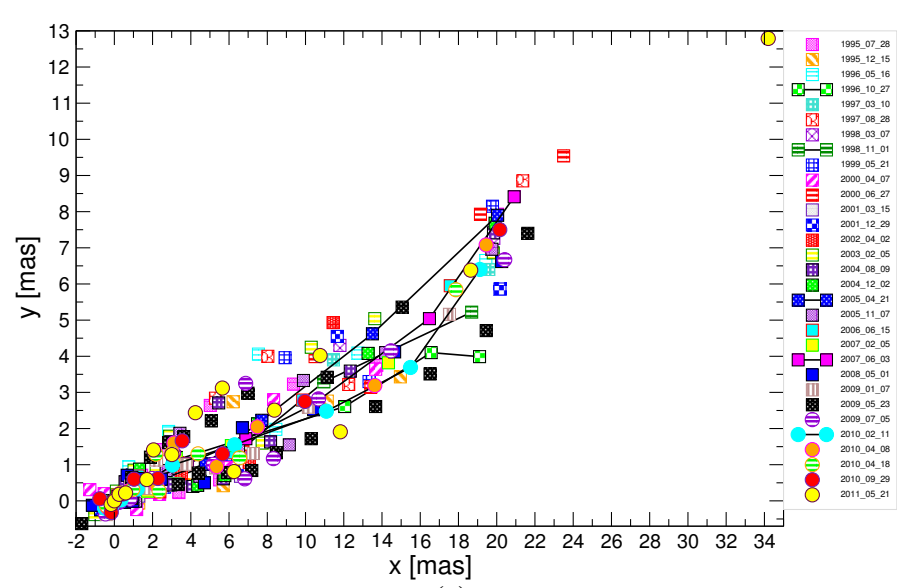

(a)

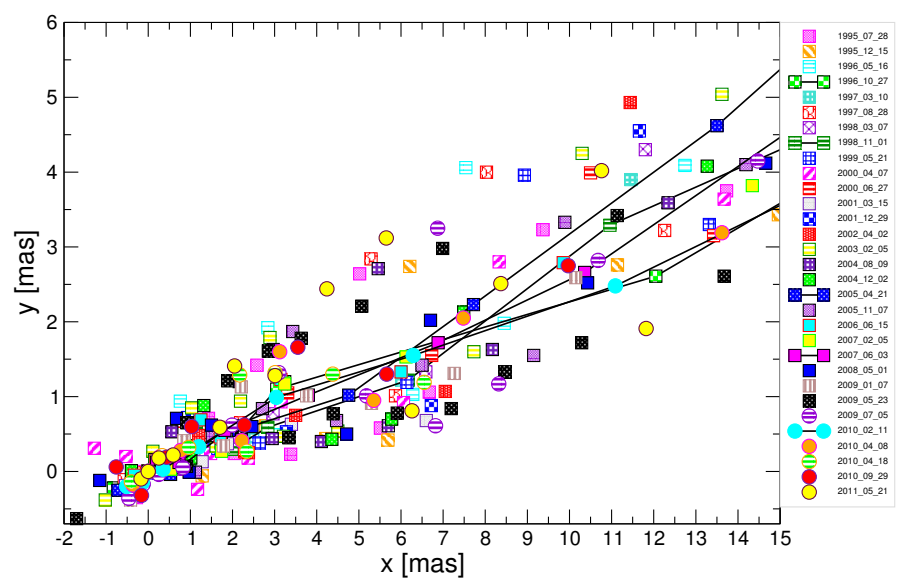

(b)

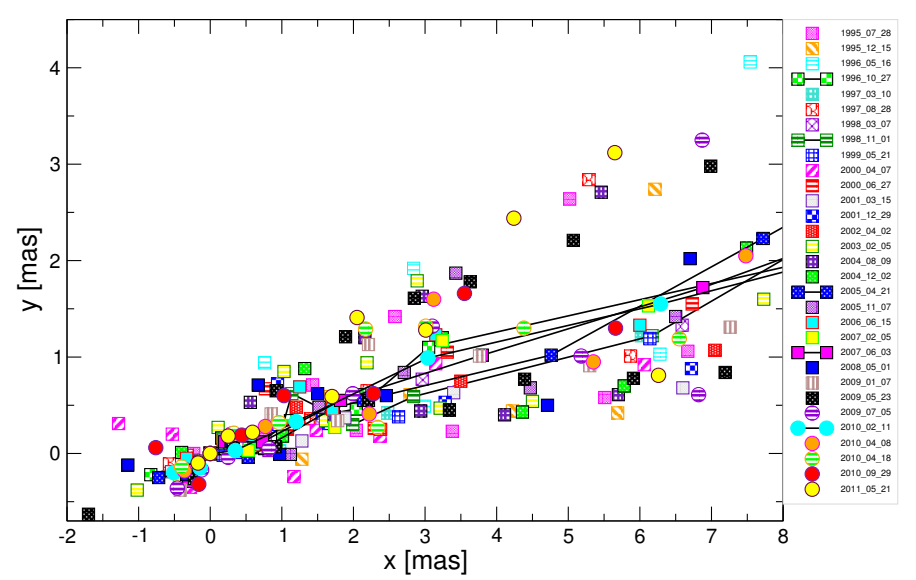

(c)

Fig. 4. Plot a) all components found in the model-fitting process in $x$ versus $y$ (upper plot). Plots b) and c) zoom into the inner 15 mas in $x \mathbf{b}$ ) and 8 mas c). All epochs are marked with different symbols and colours and the straight phases are highlighted with solid black lines.

We consider epoch 2010 because it is highly time-resolved and allows us to better identify single knots. For the counterjet we find a radial motion of 0.73 mas $\mathrm{rr}^{-1}$, whereas the jet

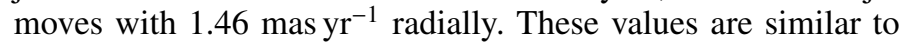
Hada et al. (2016) who find apparent velocities of $-0.63 \pm$ $0.29 \mathrm{mas} \mathrm{yr}^{-1}$ for the counter-jet ${ }^{1}$, and 1.-1.5 mas yr ${ }^{-1}$ for the jet in 2014. 1 We note that the counter-jet in the Hada et al. data seem to move
primarily not in the radial direction.

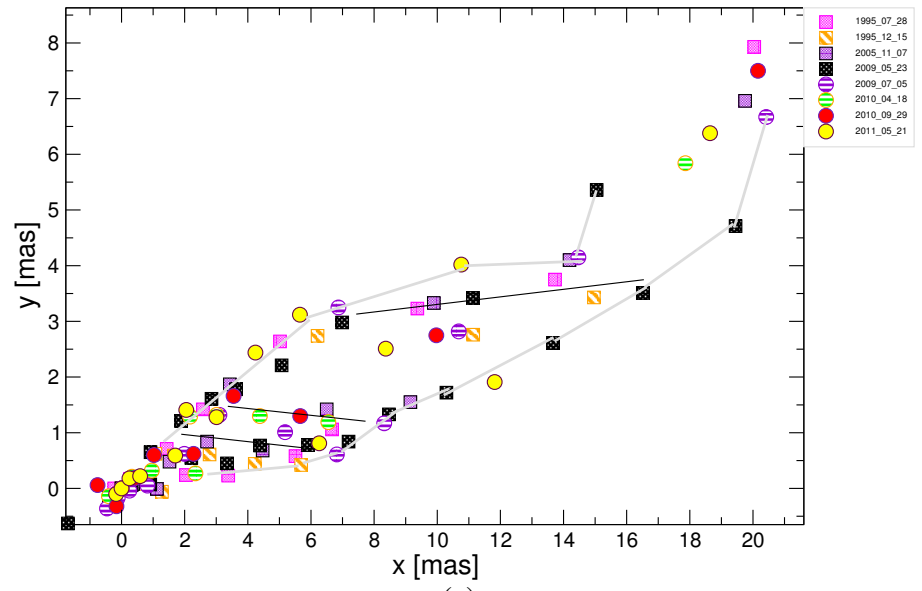

(a)

Fig. 5. Selected epochs that reveal evidence for a transverse structure. The lines are just meant to guide the eye but do not represent a fit to the data.

We applied the standard equation for superluminal jet beaming (Mirabel \& Rodriguez 1994), assuming (i) that the speed of jet and counter-jet, $\beta$, is intrinsically similar, and (ii) a fixed inclination angle between jet axis and line of sight $\theta$, such that

$\tan (\theta)=\frac{2 D}{c} \frac{\mu_{\mathrm{j}} \mu_{\mathrm{cj}}}{\mu_{\mathrm{j}}-\mu_{\mathrm{cj}}}, \quad \beta \cos (\theta)=\frac{\mu_{\mathrm{j}}-\mu_{\mathrm{cj}}}{\mu_{\mathrm{j}}+\mu_{\mathrm{cj}}}$

The radial proper motion suggested by our data is $\mu_{\mathrm{j}}=$ $1.5 \mathrm{mas} / \mathrm{yr}=2.3 \times 10^{-16} \mathrm{rad} / \mathrm{s}$ for the jet and $\mu_{\mathrm{cj}}=$ $0.73 \mathrm{mas} / \mathrm{yr}=1.12 \times 10^{-16} \mathrm{rad} / \mathrm{s}$ for the counter-jet. Equations (1) also provide an upper limit for the distance to the jet source, $D<c / \sqrt{\mu_{\mathrm{j}} \mu_{\mathrm{cj}}}$. As upper limit for the distance we find $D<61 \mathrm{Mpc}$, consistent with the true distance of $16.7 \mathrm{Mpc}$. For the inclination angle between jet and line of sight and the intrinsic jet velocity we find $\theta=37^{\circ}$ and $\beta=0.43$. These values fit to the results of Hada et al. (2016) who report viewing angles of $29^{\circ}-45^{\circ}$ and velocities of $\beta=0.29-0.50$. It is worth emphasizing that these data do not imply a highly relativistic motion of the M 87 jet, despite the apparent superluminal speed we see at other locations. As we discuss below, this geometry and alignment are too simplistic because we observe a wealth of structure besides pure radial motion.

\section{The uniqueness of M 87: probing the jet launching site of an active black hole}

\subsection{Jet launching scenarios}

Jet properties seem to be governed by accretion rate, black hole (BH) spin, magnetic field strength and configuration, and/or the properties and location of the inner accretion flow (e.g., Blandford \& Znajek 1977; hereafter: BZ; McKinney \& Gammie 2004; Tchekhovskoy et al. 2011; Blandford \& Payne 1982; hereafter: BP; Porth et al. 2011). The BZ process involves jet production by extraction of $\mathrm{BH}$ spin. These jets are Poynting flux dominated and originate in the $\mathrm{BH}$ magnetosphere. In the $\mathrm{BP}$ process, the jet originates in the accretion disk as a magnetized disk wind that is further accelerated by so-called magneto-centrifugal forces. Recent simulations (Porth \& Fendt 2010; Porth et al. 2011) demonstrate that magnetohydrodynamic 


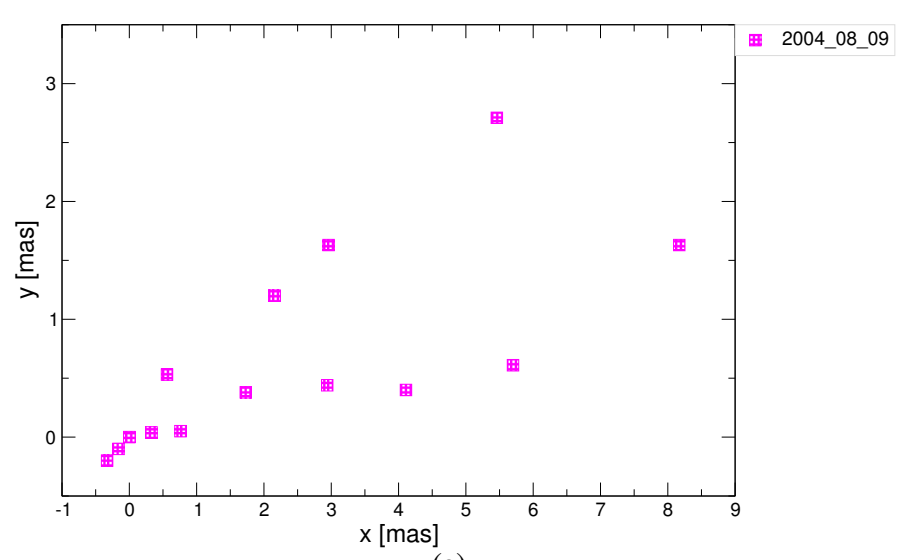

(a)

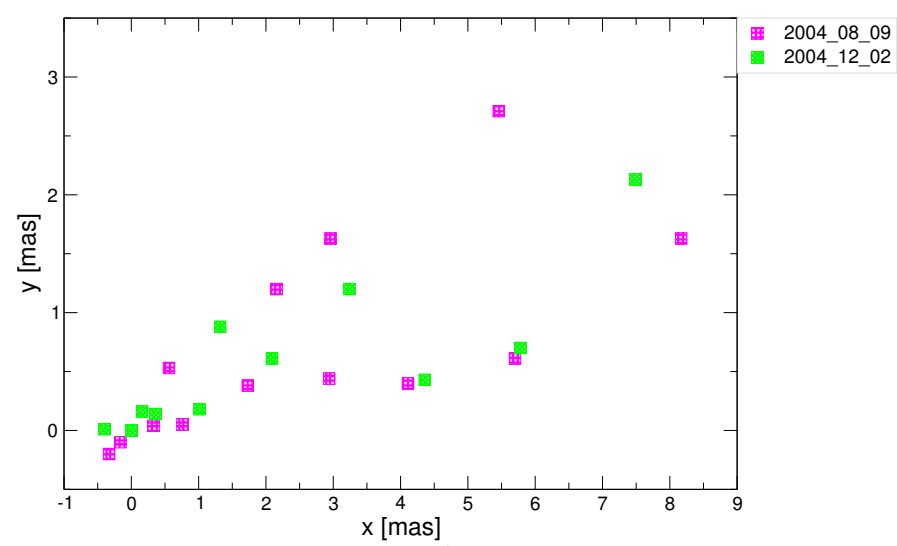

(b)

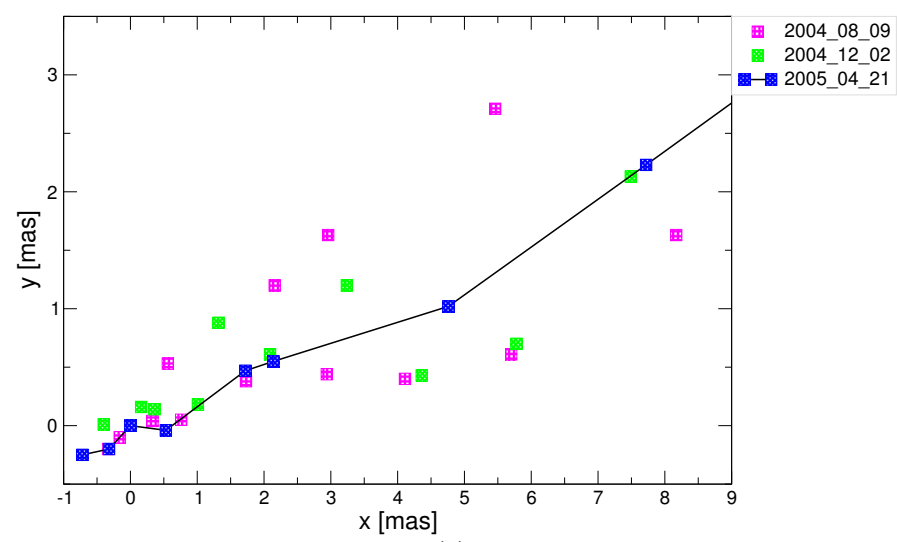

(c)

Fig. 6. Three consecutive epochs of the M 87 jet. Different colours denote for different epochs of the observations. The solid black line connects the data points of the straight phase.

(MHD) jet acceleration and collimation work similarly for relativistic and non-relativistic jets. We expect BZ jets to be more collimated right from the jet footpoint, and BP jets with a wider opening angle and collimation farther from the launching point. We expect BP outflows to be slower $(0.1 c)$ than BZ-jets. Porth et al. (2011) however show that BP jets may reach Lorentz factors of up to ten. For both BZ- and BP-jets, we expect that the radiation from jet and counter-jet should indeed be comparable right at the launching point, although this has not been investigated theoretically.

\subsection{What causes the swirling of the jet: rotation of the jet launching area?}

In Fig. 9 the end points of the lines define the starting location of the jet knots. We observe the first knots of jet and counter-jet at almost arbitrary inclinations from the jet axis (Fig. 9). During some epochs, the position of the first ejected knot seems to follow an elliptical trajectory, suggesting some kind of a swirling process.

The distance of these points from the core is about 0.3 mas on both sides. Applying the inclination angle from above, this corresponds to a deprojected distance of $1 \mathrm{pc}$ (about $100 R_{\mathrm{S}}$ ). The major axis of the ellipse is about 0.5 mas. Assuming an outflow from the very center of the core, we may understand the yet-invisible jet launching region as a bipolar cone of length and radius of $100 R_{\mathrm{S}}$, respectively, corresponding to a half opening angle of $45^{\circ}$. The potential rotation may be caused by several processes, for example (i) a precessing jet nozzle, (ii) jet knots that are localized but ejected along a jet cone and seem to be in helical motion, and (iii) ballistical jet knots ejected from a jet nozzle orbiting within the core of M 87.

A variation of jet direction over time may also indicate a precessing jet source. Precession may arise from a misaligned disk rotation axis resulting from tidal forces by the surrounding gravitational field or from a hypothetical binary component close to the jet source, indicating a central binary $\mathrm{BH}$. Similar scenarios were discussed for NGC 4258 (Cecil et al. 1992; Herrnstein et al. 2005). Precession usually happens on timescales of a few orbital periods (Sheikhnezami \& Fendt 2015) thus much longer than two years.

Indeed a precessing or conical (respectively helical) ejection is suggested by Fig. 9. The model of an orbital motion of the jet nozzle emerges if we connect the knot footpoints on both sides directly, obtaining a cylinder whose midplane forms a circle of $\sim 1 \mathrm{pc}$ radius in the plane of the core. Assuming that knots are ejected from a source orbiting along this circle, Fig. 8 suggests a timescale of about two years for an orbital motion. Combining a two year period with an orbital radius of $1 \mathrm{pc}$, we derive a central mass of $M \simeq 2 \times 10^{15} M_{\odot}$, a value that is much higher than the BH mass of M 87 and any mass that can be located in this area. We therefore exclude an orbital motion of the jet source.

Helically moving jet knots (Camenzind \& Krockenberger 1992) can explain highly variable blazar fluxes. The helical motion of jet knots results in highly variable viewing angles, leading to highly variable, Doppler-boosted knot emission. Camenzind \& Krockenberger (1992) claim that "bubbles" are injected into the large-scale jet structure resulting from "magnetic effects".

\subsection{Turbulent mass loading}

We find evidence for two modes of the jet, a "broad" phase indicated by at least two strings of knots and a "straight" phase with only one string or jet ridge line. There seems to be a transition phase wherein the jet width size is in between the broad and straight jet phase.

Interestingly, there is a close similarity between our findings and recent observations of solar jets (e.g., Moore et al. 2010; Liu et al. 2011; Pariat et al. 2015, 2016) such that solar jets also show evidence for two morphologically different phases of a straight or helical pattern (so-called standard and blowout jets). The authors present parametric studies of a three-dimensional (3D) numerical MHD model of solar-jet-like events. Both their observations and modeling results indicate that the quasi-steady 
S. Britzen et al.: A new view on the M 87 jet origin: Turbulent loading leading to large-scale episodic wiggling

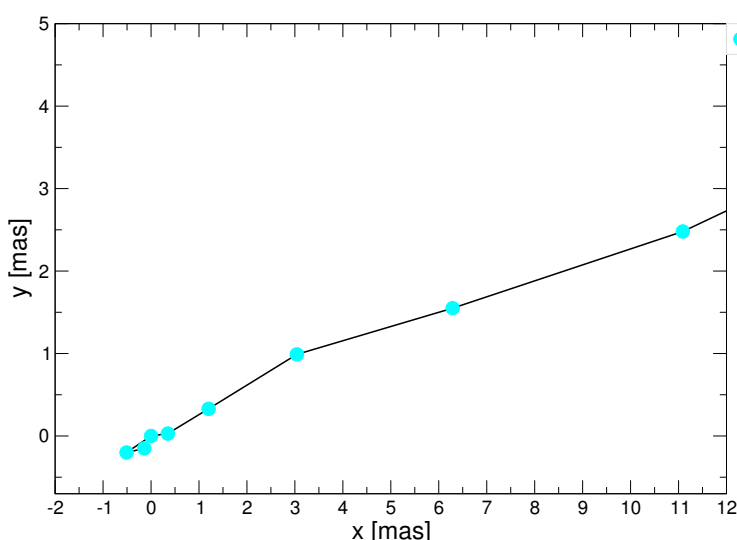

(a)

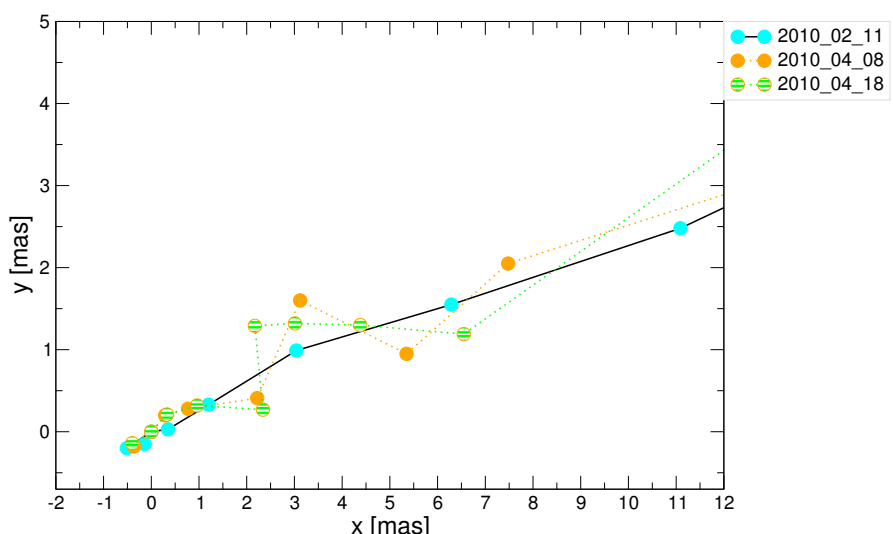

(c)

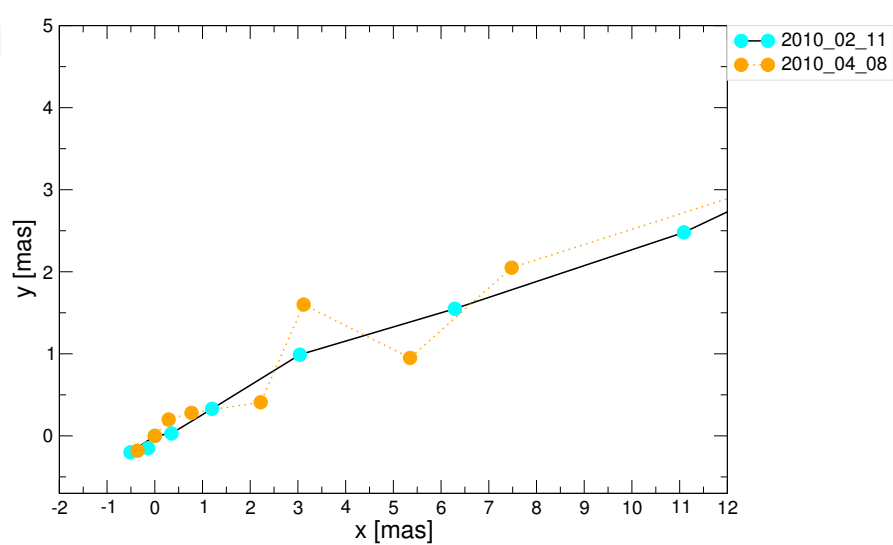

(b)

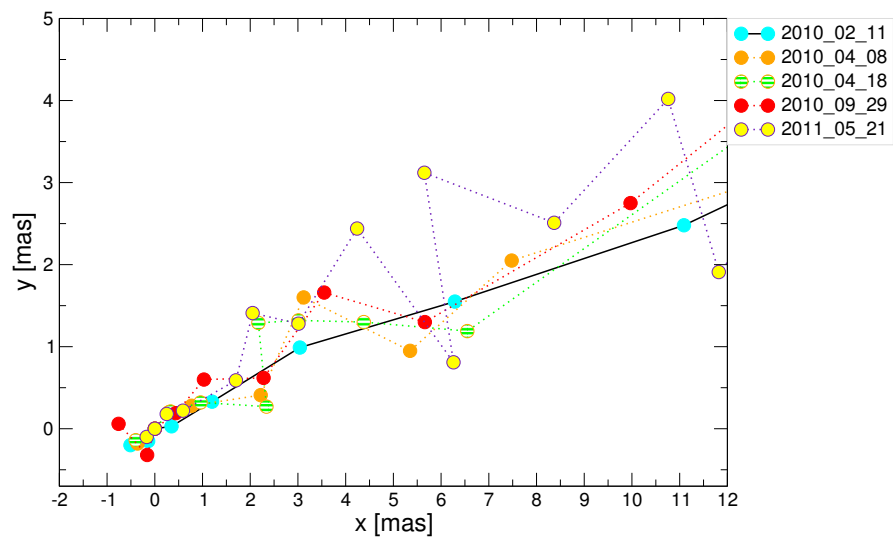

(d)

Fig. 7. Four consecutive epochs of the M 87 jet. Different colours denote for different epochs of the observations. The solid black line connects the data points of the straight epoch. The colored dotted lines connect the data points in the transition epochs.

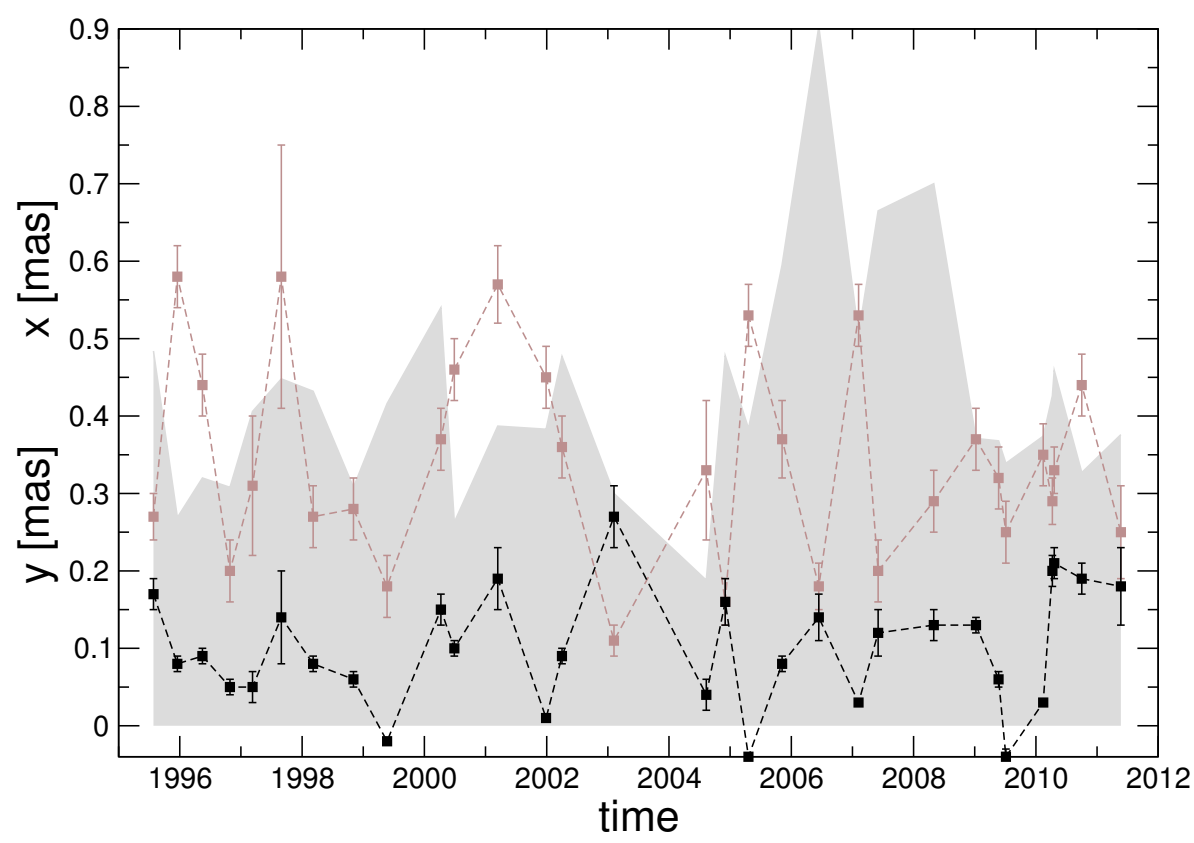

Fig. 8. Closest jet component to the core per epoch as function of time, connected by a line. Both coordinates $(x, y)$ are shown as function of time. The flux-density of the displayed components is shown in the background (gray). The $y$-axis denotes for three parameters: $x$ [mas], $y$ [mas], and fluxdensity [Jy] (not marked). reconnection phase yields a straight jet, whereas the subsequent explosive reconnection phase drives a helical jet.

With regard to our results for the M87 jet, turbulent mass loading seems reasonable, considering simulations by Romanova et al. (1998) who investigate strongly magnetized disk coronae forming from the twisted magnetic flux loops anchored in differentionally rotating parts of the accretion disk (see also Cao 2004). Indeed, we may imagine the disk surface as similar to the solar surface, which is magnetically active and shows violent reconnection events, with photospheric convection cells leading to a turbulent surface. Fendt \& Cemeljic (2002) considered jet formation including Alfvénic turbulence 


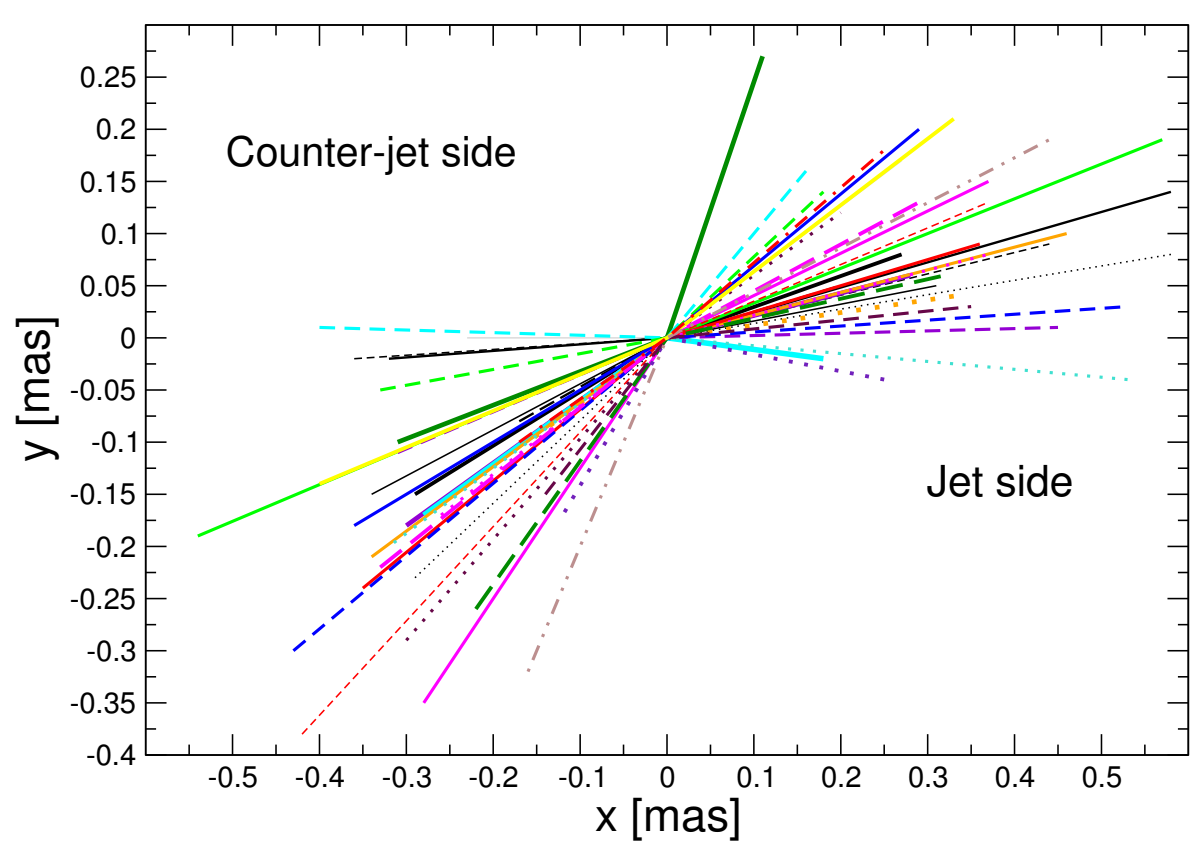

Fig. 9. Closest jet and counter-jet component to the core, per epoch. Each pair of lines is connected via the core and shown with the same color and line style.

Table 1. Proper motions and apparent speeds based on different time intervals, core distances, and jet modes.

\begin{tabular}{|c|c|c|c|c|c|c|}
\hline Epoch & Id. & $\begin{array}{l}r \\
{[\mathrm{mas}]}\end{array}$ & $\mu$ (radial) & $\mu(x, y)$ & $\begin{array}{l}\mu \text { (radial) } \\
{\left[\text { mas yr }^{-1}\right]}\end{array}$ & $\begin{array}{c}\beta_{\text {app }}(\text { radial }) \\
{[c]}\end{array}$ \\
\hline & & "Short-term" apparent motion & & & & \\
\hline \multirow[t]{4}{*}{7 Apr. 2000-27 Jun. 2000 (80 d) } & & $0.40 \pm 0.04-0.47 \pm 0.04$ & $0.07 \pm 0.07$ & $0.1 \pm 0.06$ & $0.32 \pm 0.32$ & $0.08 \pm 0.08$ \\
\hline & & $3.28 \pm 0.04-3.47 \pm 0.04$ & $0.19 \pm 0.08$ & $0.2 \pm 0.06$ & $0.87 \pm 0.37$ & $0.23 \pm 0.10$ \\
\hline & & $6.13 \pm 0.04-6.91 \pm 0.04$ & $0.78 \pm 0.08$ & $0.92 \pm 0.06$ & $3.57 \pm 0.37$ & $0.93 \pm 0.10$ \\
\hline & & $8.79 \pm 0.04-11.23 \pm 0.22$ & $2.44 \pm 0.26$ & $2.47 \pm 0.23$ & $11.16 \pm 1.19$ & $2.90 \pm 0.31$ \\
\hline \multirow[t]{4}{*}{8 Apr. $2010-18$ Apr. 2010 (10 d) } & & $0.35 \pm 0.04-0.39 \pm 0.04$ & $0.04 \pm 0.04$ & $0.04 \pm 0.05$ & $1.46 \pm 1.46$ & $0.38 \pm 0.38$ \\
\hline & & $0.82 \pm 0.04-1.01 \pm 0.04$ & $0.19 \pm 0.08$ & $0.19 \pm 0.06$ & $6.94 \pm 2.92$ & $1.80 \pm 0.76$ \\
\hline & & $2.26 \pm 0.04-2.36 \pm 0.04$ & $0.10 \pm 0.08$ & $0.19 \pm 0.06$ & $3.65 \pm 2.92$ & $0.95 \pm 0.76$ \\
\hline & & $\begin{array}{l}\text { Apparent motion in consecutive } \\
\text { epochs of a straight jet-phase }\end{array}$ & & & & \\
\hline \multirow[t]{5}{*}{27 Oct. 1996-10 Mar. 1997} & & $0.21 \pm 0.04-0.31 \pm 0.09$ & $0.10 \pm 0.10$ & & $0.27 \pm 0.27$ & $0.07 \pm 0.07$ \\
\hline & & $0.60 \pm 0.04-0.73 \pm 0.17$ & $0.13 \pm 0.13$ & & $0.35 \pm 0.35$ & $0.09 \pm 0.09$ \\
\hline & & $1.17 \pm 0.07-1.60 \pm 0.04$ & $0.43 \pm 0.11$ & & $1.16 \pm 0.30$ & $0.30 \pm 0.08$ \\
\hline & & $2.09 \pm 0.04-2.52 \pm 0.17$ & $0.43 \pm 0.21$ & & $1.16 \pm 0.57$ & $0.30 \pm 0.15$ \\
\hline & & $19.51 \pm 0.19-20.61 \pm 0.22$ & $1.1 \pm 0.41$ & & $2.97 \pm 1.11$ & $0.77 \pm 0.29$ \\
\hline \multirow[t]{4}{*}{28 Aug. 1997-7 Mar. 1998} & & $0.60 \pm 0.17-0.82 \pm 0.04$ & $0.22 \pm 0.21$ & & $0.42 \pm 0.40$ & $0.11 \pm 0.10$ \\
\hline & & $1.45 \pm 0.21-1.60 \pm 0.04$ & $0.15 \pm 0.15$ & & $0.29 \pm 0.29$ & $0.08 \pm 0.08$ \\
\hline & & $2.29 \pm 0.10-3.06 \pm 0.04$ & $0.77 \pm 0.14$ & & $1.48 \pm 0.27$ & $0.39 \pm 0.07$ \\
\hline & & $5.96 \pm 0.06-6.72 \pm 0.04$ & $0.76 \pm 0.10$ & & $1.46 \pm 0.19$ & $0.38 \pm 0.05$ \\
\hline \multirow[t]{5}{*}{5 Feb. 2007-3 Jun. 2007} & & $0.53 \pm 0.04-0.74 \pm 0.19$ & $0.21 \pm 0.21$ & & $0.66 \pm 0.66$ & $0.17 \pm 0.17$ \\
\hline & & $1.76 \pm 0.04-1.90 \pm 0.23$ & $0.14 \pm 0.14$ & & $0.44 \pm 0.44$ & $0.11 \pm 0.11$ \\
\hline & & $3.45 \pm 0.04-3.93 \pm 0.20$ & $0.48 \pm 0.24$ & & $1.50 \pm 0.75$ & $0.39 \pm 0.20$ \\
\hline & & $6.31 \pm 0.04-7.09 \pm 0.21$ & $0.78 \pm 0.25$ & & $2.44 \pm 0.78$ & $0.64 \pm 0.20$ \\
\hline & & $\begin{array}{l}\text { "Long-term" apparent motion } \\
\text { (determined via linear regression) }\end{array}$ & & & & \\
\hline 28 Jul. 1995-21 May 2011 & J6 & $5.54 \pm 0.14-11.97 \pm 0.05$ & & & $0.41 \pm 0.02$ & $0.11 \pm 0.03$ \\
\hline 16 May $1996-8$ Apr. 2010 & $\mathrm{~J} 10$ & $8.56 \pm 0.22-15.92 \pm 0.22$ & & & $0.49 \pm 0.04$ & $0.13 \pm 0.01$ \\
\hline 28 Jul. $1995-21$ May 2011 & $\mathrm{~J} 15$ & $9.91 \pm 0.35-19.70 \pm 0.14$ & & & $0.51 \pm 0.04$ & $0.13 \pm 0.01$ \\
\hline 28 Jul. $1995-29$ Sep. 2010 & $\mathrm{~J} 20$ & $19.40 \pm 0.22-23.12 \pm 0.19$ & & & $0.03 \pm 0.04$ & $0.01 \pm 0.01$ \\
\hline
\end{tabular}

in the launching region that is advected from the turbulent disk. The timescale for the decay of this turbulence is longer than the initial jet propagation timescale and thus the disk turbulence pattern can be propagated into the outflow. Putting together the turbulent structure of the inner accretion disk with flaring events of reconnecting magnetic flux tubes in the disk corona, the model of jet knots emitted as substructure into a large-scale jet magnetic field seems promising. The large-scale, aligned jet magnetic field is visible as jet structure at large distances (kpc-Mpc).

Figure 11 provides a schematic illustration of the interrelation between the turbulent injection process and the large-scale jet appearance. The turbulent mass-loading close to the disk 
S. Britzen et al.: A new view on the M 87 jet origin: Turbulent loading leading to large-scale episodic wiggling

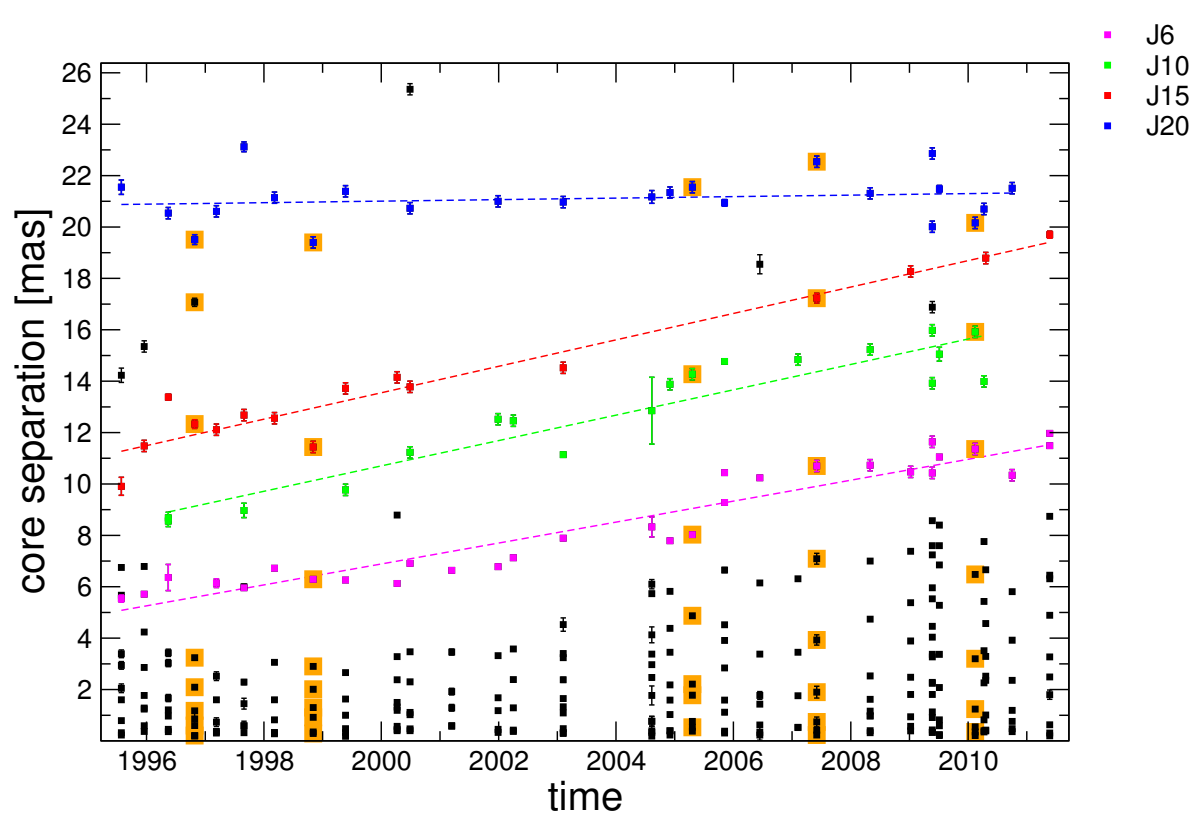

Fig. 10. All component separations as a function of time for all the 31 epochs. Identified components are marked in color. Those epochs in which a more or less straight jet ridge line is observed are marked with larger orange squares.

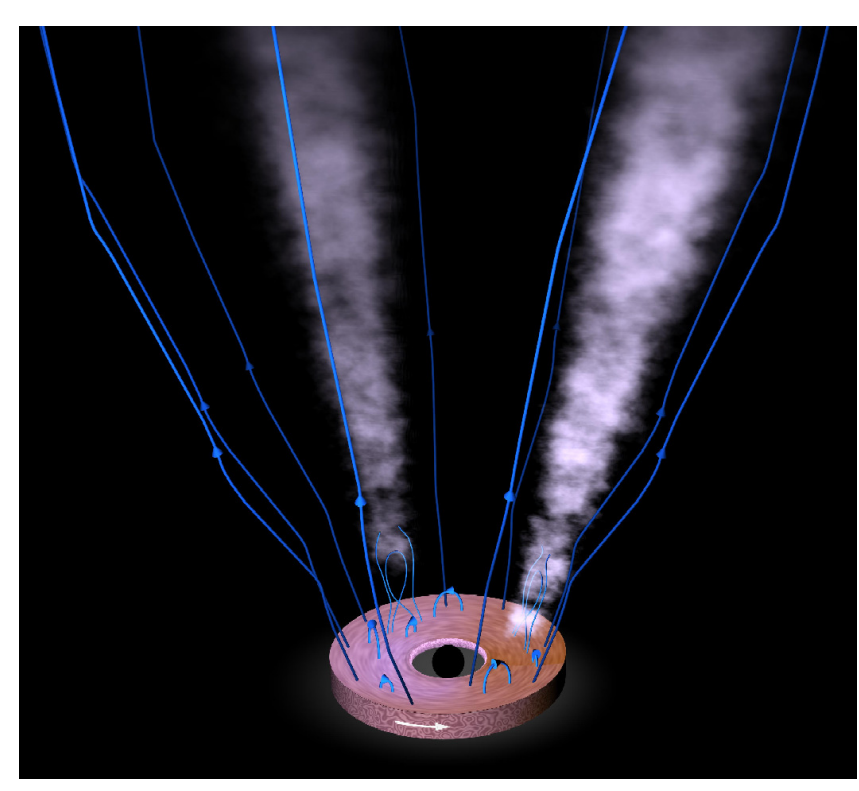

(a)

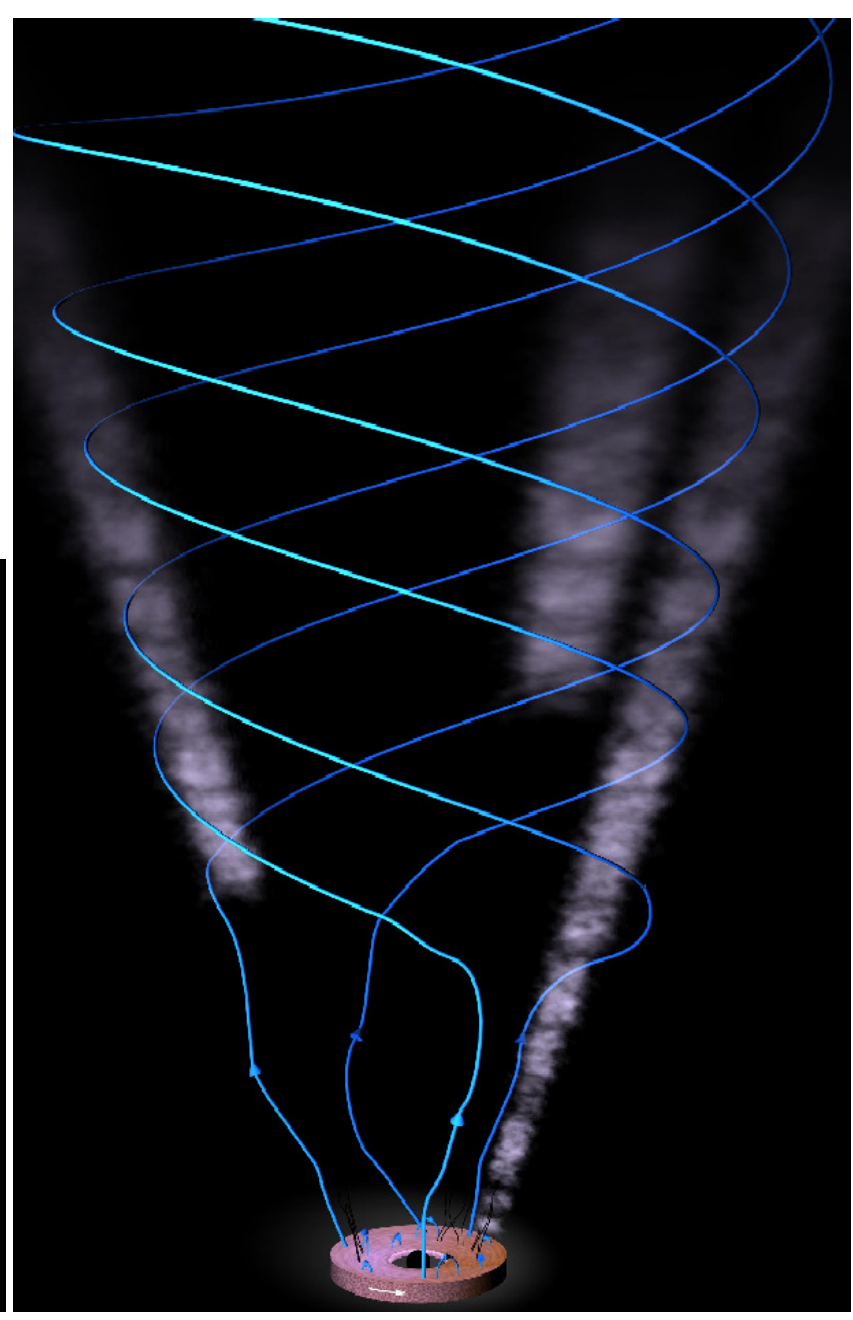

(b)

Fig. 11. Schematic illustration of the turbulent mass injection process. Turbulent mass loading close to the disk surface a) by local reconnecting magnetic flux tubes. Over long distances only the global helical magnetic field structure survives b) with a mass loading resulting from localized reconnection events from a). Figures are kindly provided by Axel. M. Quetz (graphics department of MPIA). 
surface is a local process, loading different magnetic flux tubes at different times and at different locations. The mass loading is physically done by reconnecting magnetic flux tubes originating at the disk surface. On large scales only the global field structure survives, and is turned into a helical field by the usual magneto-centrifugal driving. The mass loading of the jet, however, is not smooth, but results from the localized reconnection events on the disk surface. We note that in this picture, the mass flux has a predominantly poloidal velocity (as typical for MHD jets) whereas the magnetic field helix "rotates through" the gas distribution, satisfying the MHD condition.

A high number of studies have already explored quantitatively magnetic reconnection in the coronal region around black holes at the jet launching regions. In particular, several studies connect the magnetic power released by violent reconnection and the associated ejection of plasmons or knots along the reconnected field lines (in a Blandford-Payne scenario) with the disk accretion rate and the dimensions of the coronal region. For example, according to de Gouveia Dal Pino \& Lazarian (2005), large-scale superluminal ejections in the galactic microquasar GRS $1915+105$ during radio flare events are produced by violent magnetic reconnection episodes in the corona just above the inner edge of the magnetized accretion disk that surrounds the central $\sim 10 M_{\odot}$ black hole. According to the authors, the process occurs when a large-scale magnetic field is established by a turbulent dynamo in the inner disk region.

Yuan et al. (2009) derive ejection velocities and timescales for episodic mass loading. For Sgr $\mathrm{A}^{*}$ they find that relativistic speeds could be obtained after about $35 \mathrm{~min}$. Of course this value would strongly depend on the mass flux and the magnetic field strength, and could be very different in M 87.

Kadowaki et al. (2015) find that fast magnetic reconnection mechanisms can operate in the core regions of microquasars and low luminosity AGN (LLAGN). They test two different scenarios of fast magnetic reconnection: anomalous resistivity (AR) and turbulence. Turbulence speeds up the reconnection. Kadowaki et al. (2015) adopt the turbulent collisional fast reconnection model by Lazarian \& Vishniac (1999). The fast reconnection model investigated by Kadowaki et al. is relatively insensitive to the physical parameters inside the accretion disk, except for the accretion rate, which was allowed to vary between $0.0005 \leq \dot{m} \leq 1$.

In the case of $\mathrm{AR}$, the magnetic power released during violent fast magnetic reconnection in the surroundings of a $\mathrm{BH}$ is approximately $\dot{W}_{\mathrm{B}} \sim 2 \times 10^{39} \mathrm{erg} \mathrm{s}^{-1}$ for a $6 \times 10^{9}$ solar mass black hole accreting at Eddington rate (see their Eq. (11)). In case of the fast reconnection by turbulence $\dot{W}_{\mathrm{B}} \sim 9 \times 10^{44} \mathrm{erg} \mathrm{s}^{-1}$ is released for a $6 \times 10^{9}$ solar mass black hole (see their Eq. (15)). The turbulent model results in a larger magnetic reconnection power. Most likely though, only a fraction of this reconnection power will be effectively available to accelerate the flow. The turbulent scenario is more compatible with our results. Kadowaki et al. (2015) show a series of figures which visualize this. In their Fig. 3, they compare the magnetic power released by fast reconnection driven by $\mathrm{AR}$ and by turbulence as a function of $\mathrm{BH}$ mass. The difference is clearly visible. Their Fig. 5 shows the turbulent driven magnetic reconnection power against $\mathrm{BH}$ source mass, and M 87 fits nicely into the part which stands for a gaspressure dominated disk. Their Fig. 5 comprises the observed gamma-ray emission and Fig. 7 displays the difference of the magnetic reconnection power in M 87 and gamma-ray emitting blazars. All these plots support the fast reconnection by turbulence model for M 87.
We will discuss the relation between the radio emission and high energy emission further in Sect. 4.7. In our model, this energy generated by turbulent reconnection would be taken from the local tangled magnetic field and put into the initial acceleration of the disk wind until it will be further accelerated by the magneto-centrifugal mechanism.

Singh et al. (2015) investigate the role of fast magnetic reconnection in accreting black hole sources. Instead of assuming that the accretion disk has already evolved to a ShakuraSunyaev regime as the initial state as in previous works, they assume a magnetically dominated advective flow (MDAF) disk. The MDAF scenario naturally drives turbulence in the corona. The results by Singh et al. (2015) again indicate that fast reconnection is relatively insensitive to the accretion disk model. The similarity between the results produced in their paper with MDAF accretion and those of Kadowaki et al. (2015) with standard disk accretion is striking. The only difference is that in order for magnetic reconnection to produce both emissions, the sources in general require accretion rates of $5 \times 10^{-4}<\dot{m} \leq 0.05$ in the MDAF case and of $\dot{m}>0.05$ in the standard accretion case (Kadowaki et al. 2015).

\subsection{Jet kink instability}

We now discuss whether a jet kink instability in combination with jet rotation can lead to a time-varying bent jet structure as well. Jets are believed to carry a strong toroidal magnetic field component and are thus prone to a current-driven (CD) kink instability (Baty \& Keppens 2002). Numerical simulations of jet formation in general relativity (McKinney \& Blandford 2009) show that a helical-like kinked global jet structure can emerge. Despite this perturbation, the jet remains stable. The "wavelength" of the perturbation is some 200-300 gravitational radii (interpreting their Fig. 2). Rotation of this sort of a stable but kinked jet structure would be observed as an oscillation of the jet structure as indeed shown in our Fig. 8. Although the pure existence of a rotating kink-like structure would be compatible with the simulations of McKinney \& Blandford (2009), it is difficult to estimate typical timescales for a pattern motion of this sort of a kink perturbation. The timescale indicated by our observations is roughly two years, which would probably not be consistent with the rotational period of the black hole because we expect it to be on the order of the Keplerian speed, at approximately 10-20 Schwarzschild radii.

There are recent numerical simulations of the growth of the kink instability along rotating jets with helical fields, both for tower jets and propagating jets (Mizuno et al. 2012; Singh et al. 2016; Bromberg \& Tchekhovskoy 2016; Striani et al. 2016), which can help in estimating the typical timescales for a pattern motion to compare with our observations. These works also find evidence of turbulent reconnection driven by the kink instability.

Mizuno et al. (2012) investigated the influence of jet rotation and differential motion on the linear and nonlinear development of the CD kink instability of force-free magnetic equilibria via $3 \mathrm{D}$ relativistic MHD simulations. The key problem in GRMHD simulations of jet generation in all models is the conversion of electromagnetic energy into plasma energy. Large-scale magnetic fields may dissipate if the regular magnetic structure is destroyed as a result of a global MHD instability, the kink instability being the most plausible candidate (e.g., Begelman 1998; Giannios \& Spruit 2006). The toroidal magnetic field produced in outflows from rotating bodies (e.g., black holes, and accretion disks) becomes dominant in the far zone because the poloidal 
field falls off faster with expansion and distance. In configurations with strong toroidal magnetic field, the CD kink mode is unstable. This instability excites large-scale helical motions that can strongly distort or even disrupt the system, thus triggering violent magnetic dissipation. This instability is common in many configurations containing strong toroidal magnetic fields, such as coronal mass ejections, i.e. erupting magnetic flux ropes (e.g., Fan 2005), stellar interiors (e.g., Bonanno \& Urpin 2011), or pulsar wind nebulae (e.g., Begelman 1998).

Bromberg \& Tchekhovskoy (2016) find in time-dependent $3 \mathrm{D}$ relativistic MHD simulations of relativistic, Poynting flux dominated jets that jets are susceptible to two types of instability: i) a global, external kink mode that grows on long timescales. It bodily twists the jet, reducing its propagation velocity; and ii) a local, internal kink mode that grows over short timescales and causes small-angle magnetic reconnection and conversion of about half of the jet electromagnetic energy flux into heat. We note that these simulations have been applied for core-collapse gamma-ray burst (GRB) jets, however, the main features may also appear in other relativistic jet sources.

\subsection{Stabilizing the jet with instabilities}

The stability of jets over long distances is an essential concern, in particular because of the turbulent mass loading and the fact that they carry strong toroidal fields. In fact, the evolution of the ridge line discussed above may resemble the evolution of a kink mode.

The 3D formation of jets from disk winds have been simulated by Ouyed et al. (2003). The result is that jets maintain their long-term stability through a self-limiting process. This is accomplished in two ways: i) a strong poloidal magnetic field is concentrated along the central axis of the jet constituting a "backbone" in which the Alfvén speed is sufficiently high to reduce the average jet Alfvénic Mach number to unity; and ii) the onset of higher-order Kelvin-Helmholtz modes reduces the efficiency with which the jet material is accelerated, and transfer kinetic energy of the outflow into the stretched, poloidal field lines of the distorted jet. Another interesting result of these studies is that jets go into alternating periods of low and high activity because the disappearance of unstable modes in the sub-Alfvénic regime enables another cycle of acceleration to super-Alfvénic speeds. In the process of taming the effects of the kink mode, jets settle into relatively stable end-states involving either corkscrew (their lower panel in Fig. 2), or wobbling types of structure (their upper four panels in Fig. 2). In the case of the M 87 jet, we find different morphologies: a turbulent phase within the inner 5 mas, and a more ordered behavior of component motion from 6 mas outward and at larger separations indications for helical structures.

The scenario presented by Ouyed et al. (2003) is similar to the simulations by McKinney \& Blandford (2009). A helical kink co-rotating with the accretion disk may in principle explain the helical structure of the M87 jet at intermediate distances. The angular velocity of the helical structure is comparable to the disk rotation, but also depends on the launching radius of the jet. This velocity corresponds to a rotational period of hours or days, which is much less than the timescale of two years seen in our data. We should therefore exclude the hypothesis of a rotating instability pattern as an explanation. On the other hand, if this sort of a helical kink is caused by an instability during jet formation, as we observe on larger distance, the jet substructure could be a result of the mass-loading process. We propose here that this process is turbulent in nature and finally results in filling-in and brightening different channels (flux tubes) of the overall (helical) jet flow.

\subsection{The overall picture}

Interestingly, the different physical processes discussed above are potentially interrelated and can be seen at work in our data of the M87 jet. In magnetically dominated regions, especially near the nuclear region, shocks are faint and particle acceleration is attributed to the Fermi process in magnetic reconnection discontinuities (de Gouveia Dal Pino \& Lazarian 2005). When jets are magnetically dominated, they are likely to experience current driven instabilities, which in turn may drive reconnection. Twisted structures and the sense of twist can be triggered by the precession of the jet base (e.g., Begelman et al. 1980), by rotation of the jet fluid, or by magnetic field helicity.

In their study, Singh et al. (2016) impose a precessional perturbation at the inlet of a rotating cylindrical jet. This leads to the displacement of the helical magnetic field configuration and induces the CD kink instability, which in turn leads to magnetic reconnection and energy dissipation. This energy goes to the kinetic energy of the flow and may also accelerate particles through a first-order Fermi process in the magnetic reconnection regions (e.g., de Gouveia Dal Pino \& Lazarian 2005).

Striani et al. (2016) investigate 3D MHD simulations of magnetic reconnection in a magnetically confined cylindrical plasma column. They find that the 3D instabilities acting on these configurations result in a fragmentation of the initial current sheet into small filaments, leading to an enhanced dissipation rate.

Singh et al. (2016) investigate the influence of the radial density profile on the spatial development of the CD kink instability along magnetized, rotating, relativistic jets. The results show the propagation of a helically kinked structure along the jet and a relatively stable configuration for the lighter jets. The jets appear to be collimated by the magnetic field, and the flow is accelerated owing to conversion of electromagnetic into kinetic energy. They identify regions of high current density in filamentary current sheets, indicative of magnetic reconnection. These sorts of sheets are associated with the kink-unstable regions and correlate with the decrease of the sigma parameter of the flow. They find that fast magnetic reconnection may be driven by the kinkinstability turbulence and govern the transformation of magnetic into kinetic energy, thus providing an efficient way to power and accelerate particles in AGN.

Do our data show a preference for either the BZ or the BP process? The relatively low speed together with a wide opening angle, and the limb-brightened structure with a seemingly hollow beam, indicate a BP launching of the M 87 jet. A helical jet structure with a rotational feature may arise in both scenarios. A periodicity of about two years indicated by our observations is larger than what we expect from a $\mathrm{BH}$ rotational velocity in M 87, assuming maximum rotation. The (bulk) rotation could be a pattern velocity and not be related to the rotation of an underlying source. Most probably, both launching processes play a role.

Higher resolution observations will allow us to address the question of whether the jet base is located exactly on the rotation axis of the $\mathrm{BH}$, or whether it rises from the equatorial plane at some distance (a few Schwarzschild radii). This question addresses the problem of whether frame-dragging plays a role in triggering the magnetic reconnection, and whether this happens within the BH ergosphere or outside (e.g., Koide \& Arai 2008; Karas et al. 2012). 


\subsection{Turbulence and the M87 TeV emission}

A long-standing question with regard to AGN is the origin of the TeV-emission. So far, AGN with highly-beamed jets toward the line of sight have been the largest sample of sources detected at very high energies. A few nearby radio galaxies, considered to be low luminosity AGN (LLAGN), have been detected at TeV energies (HEGRA, HESS, MAGIC and VERITAS) as well. The radio galaxy M 87 is among those detections but does not fit into the category of highly-beamed sources because the estimated viewing angle for M 87 is on the order of $30^{\circ}$ (as discussed in this paper) and allows only moderate Doppler boosting.

In addition, the AGN source in M87 is highly underluminous and the $\mathrm{TeV}$ emission in M 87 is highly variable with timescales of a few days $\left(t_{\mathrm{var}} \sim 1-2\right.$ days), pointing to extremely compact emission regions corresponding to scales of a few Schwarzschild radii (e.g., Abramowski et al. 2012). When driven by turbulence, not only the radio but also the gammaray emission can be due to magnetic power released by fast reconnection, which according to Kadowaki et al. (2015) may accelerate particles to relativistic velocities in the core region of LLAGN such as M 87. They find that the corresponding gammaray emission of LLAGN can be associated with the same emission zone that produces the radio synchrotron emission in the core of these sources.

The observed gamma-ray luminosity of these sources is almost correlated with both the radio luminosity and the calculated magnetic reconnection power according to Kadowaki et al. (2015). It thus seems possible, that the particles accelerated by the magnetic reconnection mechanism can produce the radio emission and can also be responsible for the processing of the high energy emission in the core region. This fits nicely with the results by Wagner et al. (2009). From VLBA monitoring observations at $43 \mathrm{GHz}$ that were made during the same period of the VERITAS, MAGIC and H.E.S.S. observations, a temporal coincidence of $\mathrm{TeV}$ and radio flares could be deduced in the case of M 87, indicating that they are related (Wagner et al. 2009). According to the authors this provides the first direct evidence that the $\mathrm{TeV}$ radiation from this source is produced within a few tens of Schwarzschild radii of the radio core.

Kadowaki et al. (2015) find considerably different results in the case of blazars. The emission in blazars cannot be attributed to the same process. Their result is consistent with the standard scenario for blazars where the emission is attributed to relativistic particle acceleration along the jet which has relativistic bulk velocities. Any deep core emission in these sources is probably screened by opacity and the jet pointing toward our line of sight.

This underlines one of the basic results of our paper. In M 87 we have the chance to look deeper into the core region and decipher the energy production in much better detail. The M 87 jet visible at $15 \mathrm{GHz}$ probes a different physical zone compared to the standard blazar-zone we tend to see in AGN jets.

\section{Discussion}

In general, AGN pc-scale jets are observed, monitored, and studied in a region far away from the launching and collimation site (e.g., CJF: Britzen et al. 2008; MOJAVE: Lister et al. 2009). The kinematic information derived for the M 87 jet differs in many aspects from typical AGN jets. In the following, we discuss the main findings of our observations in comparison.

The sampling rate of the MOJAVE survey is not high enough to fully cover the different motions in the M87 jet. We find fast apparent motions from epochs that are close in time, and evidence for acceleration along the jet. We test several scenarios to determine the apparent motions. It seems possible to determine the apparent velocities of the jet components in the straight phases. However, because the time resolution is not sufficient we cannot be sure that we can cover the motion in full detail.

The M 87 jet is limb-brightened in most of the epochs but motion does not seem to occur along the two limbs. Instead we find evidence for different modes or episodes that reveal either a jet with several strings (limbs) or an almost straight jet with only one string of jet ridge line. In addition, there is evidence for a transition phase between these two jet modes in which the two-stringed jet appears more narrow. There is also evidence for transverse structure along the jet. The transverse or zigzag-pattern could be explained as helical paths in projection. The mode changes convince us that we observe some form of jet oscillation. Jet oscillations are likely to be caused or injected via the accretion disk or accretion disk corona. A classical component identification, meaning a standard procedure pc-scale jet analysis, is thus significantly aggravated.

In a standard quasar jet, a component is understood as a feature that can be traced over time and is clearly separated from other components by moving on a well-defined path. In M 87 a component seems to be the result of an evolutionary sequence of the jet ridge line, which only exists temporarily and will be built up and destroyed at a different place and time along the jet in accordance with a pattern. We identify structures which together may constitute one component at larger core separations (J6, J10, J15, J20 in Fig. 10). We thus may in fact witness the formation of components in a jet.

Jet features seem to be injected in different directions into the jet. The individual epochs' jet opening angles can be much smaller (corresponding to first component size). Both jet-sides reveal a similarly broad opening angle.

We find evidence of a swirling origin of the jet. As a plausible scenario, we refer to Romanova et al. (1998) who investigate the evolution of magnetized disk coronae consisting of twisting magnetic flux loops. We understand that the accretion disk surface is similar to the solar surface in that it is turbulent, convective, and magnetically active showing violent reconnection events (Yuan et al. 2009). We therefore suggest a model of jet formation in which jet knots are injected as turbulent substructure into a large-scale jet. We provide two schematic figures to illustrate the process in Fig. 11.

As an alternative model for the jet swirling that we observe, a rotating kink instability, such as shown numerically by McKinney \& Blandford (2009), may be considered. However, it is difficult to estimate typical timescales for a pattern motion and compare them to our observations.

Our findings are supported by independent variability studies. Avachat et al. (2014) show, based on a high resolution polarimetry and variability study of VLA and Hubble Space Telescope (HST) data, that the jet's morphology in total and polarized light changes significantly on timescales of about a decade. Perlman et al. (2011) find, based on optical polarization and spectral variability in the M 87 jet, evidence that the variability in the nucleus can best be understood as either a helical distortion to a steady jet in which the distortion would arise from kink mode instabilities, or fluctuations in the jet speed that produce corresponding fluctuations in the strength of shocks within the nuclear jet. The most likely mechanism according to them is a CD-instability combined with a fluctuating, helical magnetic pitch angle.

Independent support for our results comes from highest energy observations of M87. Wagner et al. (2009) provide 
evidence that the TeV-emission of M 87 is produced within a few tens of $R_{\mathrm{S}}$ of the radio core. According to Kadowaki et al. (2015) the $\mathrm{TeV}$-emission could result from the same magnetic reconnection process as the radio emission.

A long-standing question with regard to AGN kinematics is whether the only difference between BL Lacs and Quasars is the viewing angle, with the BL Lac objects being more aligned. Britzen et al. (2010) showed that the kinematics in BL Lac Object jets differ significantly from those in a Quasar jet. The jet components in $1803+784$ predominantly reveal motion perpendicular to the jet - a displacement of the jet axis. Here, we find some resemblance to the kinematics in M 87 that supports the idea by Tsvetanov et al. (1998) that M 87 is a misaligned BL Lac object. This requires further observations and studies, and $1803+784$ could allow us to study those kinematic effects that M 87 would reveal when observed at larger separations from the core.

\section{Summary}

We shed light on a long-standing problem regarding contradictory apparent speeds in the pc-scale jet of M 87. We find an indication for apparent superluminal motion and detect acceleration in the pc-scale jet at distances up to 10 mas. Most likely the apparent and well-known limb-brightened structure is a result of jet oscillations being caused by MHD instabilities that originate in reconnection processes in the accretion disk (or corona). We find apparent mode changes of the jet, such that phases of a broad (two-string) jet alternate with comparatively seldom phases of a straight jet. The core region is particularly affected by the oscillations and a swirling of the jet origin, leading to a fairly broad and similarly sized opening angle on the jet and counter-jet sides. The traditional concepts of apparent jet component motion, jet component ejections, or viewing angle may have to be revisited in the light of the results presented here. Our results indicate that the kinematics is dominated by turbulent processes most likely resulting from reconnection processes in the accretion disk (or corona). This scenario is supported by the Blandford \& Paynemodel, although we cannot rule out a Blandford \& Znajek origin or contribution. As a backbone structure, a global magnetic field is indispensable.

The current work demonstrated the potential to explore the physics at the launching site of this prototypical object in more detail with improved resolution (GMVA+phased ALMA observations at $3 \mathrm{~mm}$ and EHT observations at $1 \mathrm{~mm}$ ) and with regard to the theoretical and simulation perspectives. Modeling of the physical processes involved within the launching process is in preparation.

Acknowledgements. We appreciate the important and motivating advice from the anonymous referee, which helped to improve the manuscript significantly. We thank G. Dvali for inspiring and enlightening discussions. We are thankful for very helpful discussions with F. Yuan and R. Keppens. The help of A. M. Quetz and the graphics department of the MPIA are appreciated for providing the artistic illustration. This research has made use of data from the MOJAVE database that is maintained by the MOJAVE team (Lister et al. 2009, AJ, 137, 3718).

\section{References}

Abramowski, A., Acero, F., Aharonian, F., et al. 2012, A\&A, 746, A151 Asada, K., Nakamura, M., Doi, A., et al. 2014, ApJ, 781, 2

Avachat, S. S., Perlman, E. S., Sparks, W. B., et al. 2014, Proc. IAU Symp., 313 Baty, H., \& Keppens, R. 2002, ApJ, 580, 800
Begelman, M. C. 1998, ApJ, 493, 291

Biretta, J. A., \& Junor, W. 1995, Proc. National Academy of Sciences of the United States of America, 92, 11364

Biretta, J. A., Zhou, F., \& Owen, F. N. 1995, ApJ, 447, 582

Biretta, J. A., Sparks, W. B., \& Macchetto, F. 1999, ApJ, 520, 621

Blandford, R. D., \& Payne, D. G. 1982, MNRAS, 199, 883 Blandford, R. D., \& Znajek, R. L. 1977, MNRAS, 179, 433

Bonanno, A., \& Urpin, V. 2011, A\&A, 525, A100

Britzen, S., Vermeulen, R. C., Campbell, R. M., et al. 2008, A\&A, 484, 119

Britzen, S., Kudryavtseva, N. A., Witzel, A., et al. 2010, A\&A, 511, A57

Broderick, A. E., Loeb, A., \& Reid, M. J. 2011, ApJ, 735, 57

Bromberg, O., \& Tchekhovskoy, A. 2016, MNRAS, 456, 1739

Camenzind, M., \& Krockenberger, M. 1992, A\&A, 255, 59

Cao, X. 2004, ApJ, 613, 716

Cecil, G., Wilson, A. S., \& Tully, R. B. 1992, ApJ, 390, 365

Cheung, C. C., Harris, D. E., \& Stawarz, L. 2007, ApJ, 663, 65

de Gouveia Dal Pino, E. M., \& Lazarian, A. 2005, A\&A, 441, 845

Dodson, R., Edwards, P. G., \& Hirabayashi, H. 2006, PASJ, 58, 243

Fan, Y. 2005, ApJ, 630, 543

Fendt, C., \& Cemeljic, M. 2002, A\&A, 395, 1045

Fish, V. L., Akiyama, K., Bouman, K. L., et al. 2016, Galaxies, 4, 54

Ford, H. C., Harms, R. J., Tsvetanov, Z. I., et al. 1994, ApJ, 435, 27

Giannios, D., \& Spruit, H. C. 2006, A\&A, 450, 887

Giroletti, M., Hada, K., Giovannini, G., et al. 2012, A\&A, 538, A10

Hada, K., Kino, M., Doi, A., et al. 2016, ApJ, 817, 131

Herrnstein, J. R., Moran, J. M., Greenhill, L. J., \& Trotter, A. S. 2005, ApJ, 629, 719

Junor, W., \& Biretta, J. A. 1995, AJ, 109, 500

Kadowaki, L. H. S., de Gouveia Dal Pino, E. M., \& Singh, C. B. 2015, ApJ, 802, 113

Karas, V., Kopacek, O., \& Kunneriath, D. 2012, Classical and Quantum Gravity, 29, 035010

Kim, J. Y., Lu, R.-S., Krichbaum, T. P., et al. 2016, Galaxies, 4, 39

Koide, S., \& Arai, K. 2008, ApJ, 682, 1124

Kovalev, Y. Y., Lister, M. L., Homan, D. C., \& Kellermann, K. I. 2007, ApJ, 668, 27

Lazarian, A., \& Vishniac, E. T. 1999, ApJ, 517, 700

Lister, M., Aller, H. D., Aller, M. F., et al. 2009, AJ, 137, 3718

Liu, C., Deng, N., Liu, R., et al. 2011, ApJ, 735, L18

Lu, R.-S., Broderick, A. E., Baron, F., et al. 2014, ApJ, 788, 120

Ly, C., Walker, C. R., \& Junor, W. 2007, ApJ, 660, 200

McKinney, J. C., \& Blandford, R. D. 2009, MNRAS, 394, 126

McKinney, J. C., \& Gammie, C. F. 2004, ApJ, 611, 977

Mertens, F., Lobanov, A. P., Walker, R. C., \& Hardee, P. E., 2015, A\&A, 595, A54

Mirabel, I. F. \& Rodriguez, L. F. 1994, Nature, 371, 46

Mizuno, Y., Lyubarsky, Y., Nishikavwa, K.-I., \& Hardee, P. 2012, ApJ, 757, 16

Moore, R. L., Cirtain, J. W., Sterling, A. C., \& Falconer, D. A. 2010, ApJ, 720, 757

Ouyed, R., Clarke, D. A., \& Pudritz, R. E. 2003, ApJ, 582, 292

Owen, F. N., Hardee, P. E., \& Cornwell, T. J. 1989, ApJ, 340, 698

Pariat, E., Dalmasse, K., DeVore, C. R., et al. 2015, A\&A, 573, A130

Pariat, E., Dalmasse, K., DeVore, C. R., et al. 2016, A\&A, 596, A36

Perlman, E. S., Adams, S. C., Cara, M., et al. 2011, ApJ, 743, 119

Porth, O., \& Fendt, C. 2010, ApJ, 709, 1100

Porth, O., Fendt, C., Meliani, Z., \& Vaidya, B. 2011, ApJ, 737, 42

Reid, M. J., Biretta, J. A., Junor, W., et al. 1989, ApJ, 336, 112

Romanova, M. M., Ustyugova, G. V., Koldoba, A. V., Chechetkin, V. M., \& Lovelace, R. V. E. 1998, ApJ, 500, 703

Sheikhnezami, S., \& Fendt, C. 2015, ApJ, 840, 113

Shepherd, M. C. 1997, Astronomical Data Analysis Software and Systems VI, ASP Conf. Ser., 125, 77

Singh, C. B., de Gouveia Dap Pino, E. M., \& Kadowaki, L. H. S. 2015, ApJ, 799, 20

Singh, C. B., Mizuno Y., \& de Gouveia dal Pino E. M. 2016, ApJ, 824, 48

Striani, E., Mignone, A., Vaidya, B., Bodo, G., \& Ferrari, A. 2016, MNRAS 462, 2970

Tchekhovskoy, A., Narayan, R., \& McKinney, J. C. 2011, MNRAS, 418, 79

Tsvetanov, Z. I., Hartig, G. F., Ford, H. C., et al. 1998, ApJ, 493, 83

Wagner, R. M., Beilicke, M., Davies, F., Hardee, P., et al. 2009, Proc. Fermi Symposium, eConfProceedings C091122

Walker, R. C., Ly, C., Junor, W., \& Hardee, P. J. 2008, J. Phys. Conf. Ser., 131, 012053

Wilson, A. S., \& Yang, Y. 2002, ApJ, 568, 133

Yuan, F., Lin, J., Wu, K., \& Ho, L. C. 2009, MNRAS, 395, 2183 


\section{Appendix A: Additional figures}

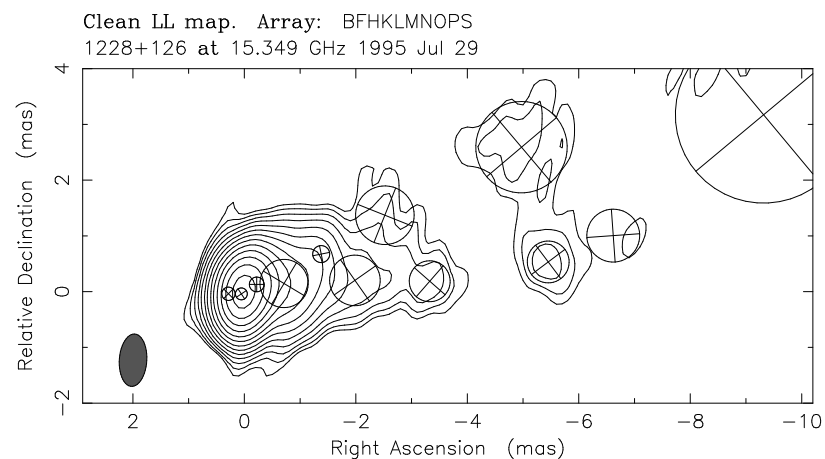

Map center: RA: 1230 49.423, Dec: +122328.044 (2000.0)

Map peak:

Contours \%: $0.60 .91 .352 .033 .04 \quad 4.566 .8310 .3$

Contours \%: 15.423 .134 .651 .977 .8

Beam FWHM: $0.941 \times 0.493$ (mas) at $-3.67^{\circ}$
Clean LL map. Array: BFHKLMNOPS

$1228+126$ at $15.353 \mathrm{GHz} 1995 \mathrm{Dec} 15$

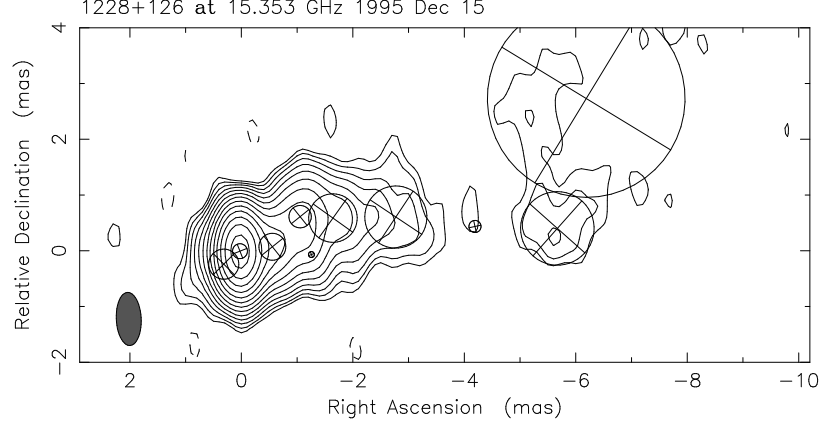

Map center: RA: 1230 49.423, Dec: +122328.044 (2000.0)

Map peak: $1.01 \mathrm{Jy} /$ bean

Contours \%: $-0.60 .60 .91 .352 .03 \quad 3.044 .566 .83$

Contours \%: 10.315 .423 .134 .651 .977 .8

Beam FWHM: $0.954 \times 0.447$ (mas) at $2.63^{\circ}$

$1228+126$ at $15.363 \mathrm{GHz} 1996 \mathrm{May} 18$

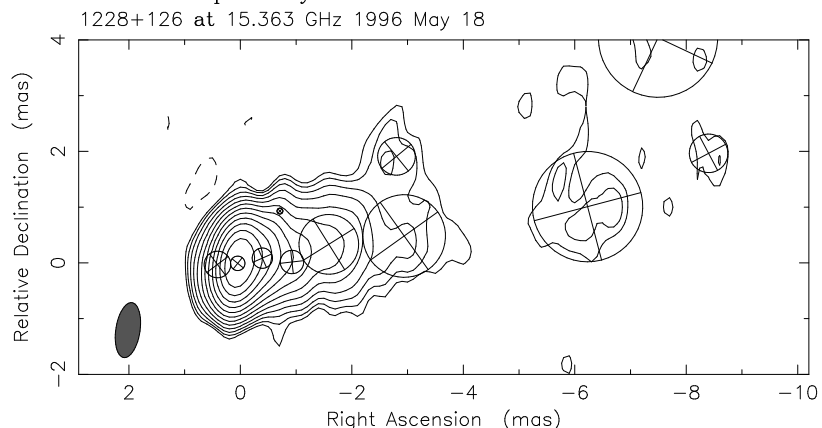

Map center: RA: 123049.423

Contours \%: -0.70 .71 .051 .572 .363 .545 .327 .97$

Contours \%: 1217.926 .940 .460 .5

Beam FWHM: $0.996 \times 0.435$ (mas) at $-8.65^{\circ}$

LL map. Array: BFHKLMNOPS

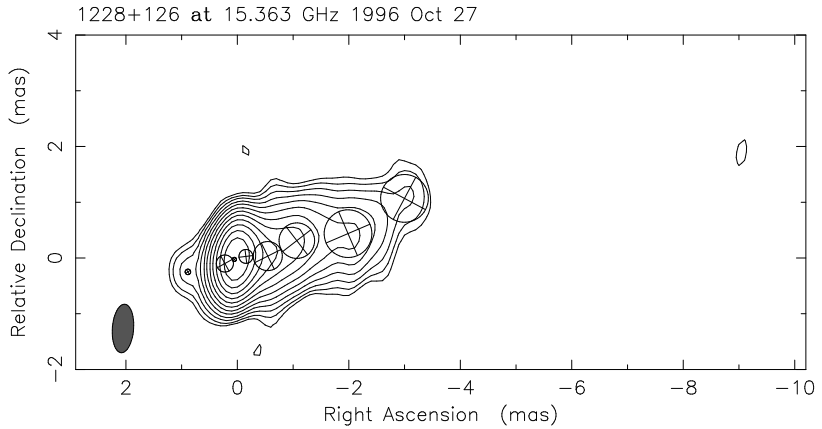

Map center: RA: 123049.423 , Dec: +12 2328.044 (2000.0)

Map peak: $0.701 \mathrm{Jy} /$ beam

Contours \%: 0.71 .051 .572 .363 .545 .327 .9712

Contours \%: 17.926 .940 .460 .5

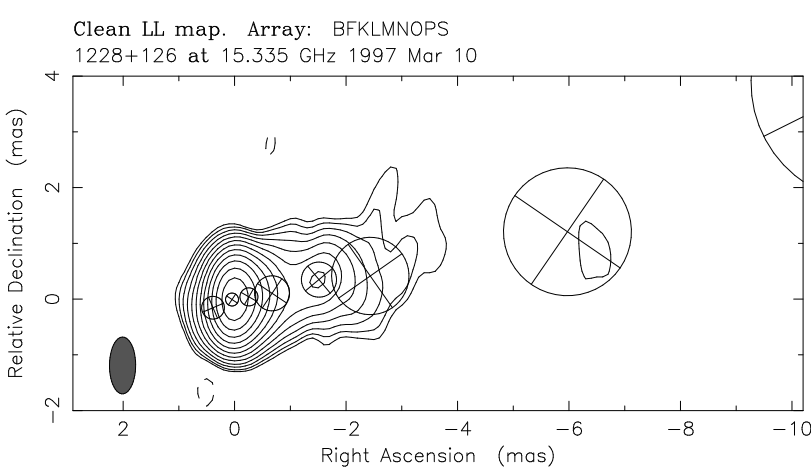

Map center: RA: 1230 49.423, Dec: +12 2328.044 (2000.0)

Map peak: $1.06 \mathrm{Jy} /$ beam

Contours \%: -0.80 .81 .21 .82 .74 .056 .089 .11$

Contours \%: 13.720 .530 .846 .169 .2

Beam Fin: $1.02 \times 0.47$ (mas) at $-0.225^{\circ}$

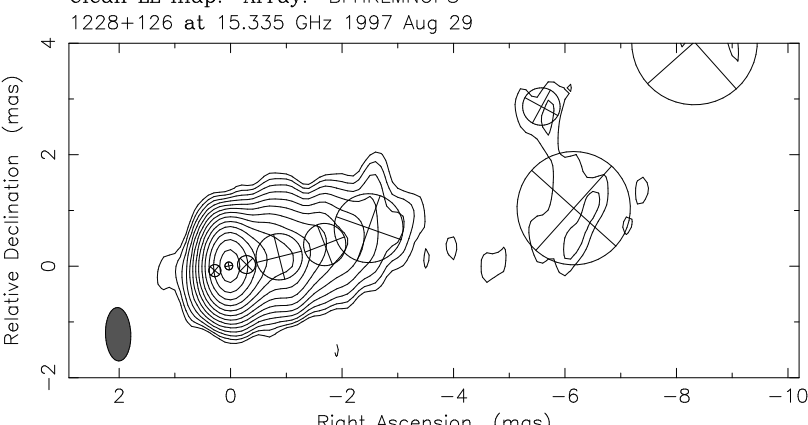

Map center: RA: 1230 49.426, Dec: +12 2328.119 (2000.0)

Map peak: $0.96 \mathrm{Jy} /$ beam

0.60 .60 .91352 .033 .044 .566 .83

Contours \%: 10.315 .423 .134 .651 .977 .8

Clean Lt $0.959 \times 0.454$ (mas) at $2.03^{\circ}$ OP

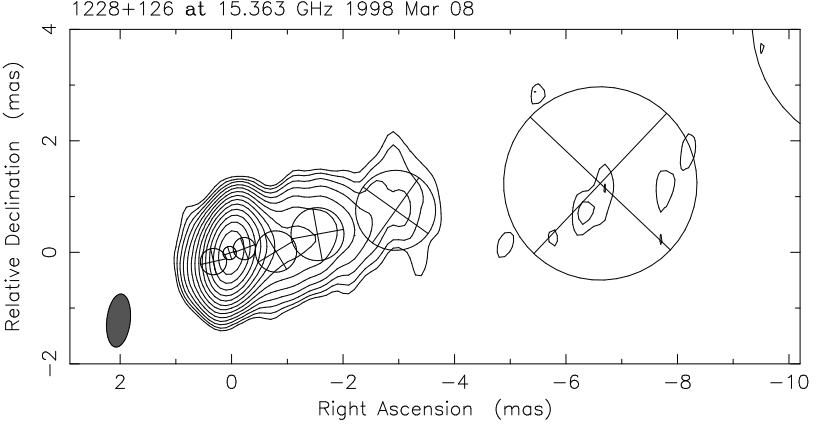

Map center: RA: 1230 49.426, Dec: +12 2328.119 (2000.0)

Map peak: $1.18 \mathrm{Jy} /$ beam

Contours \%: $0.5 \quad 0.75 \quad 1.12 \quad 1.692 .53 \quad 3.85 .78 .54$

Contours \%: 12.819 .228 .843 .264 .9

Beam Finm: $0.057 \times 0.422$ (mas) at $-6.39^{\circ}$

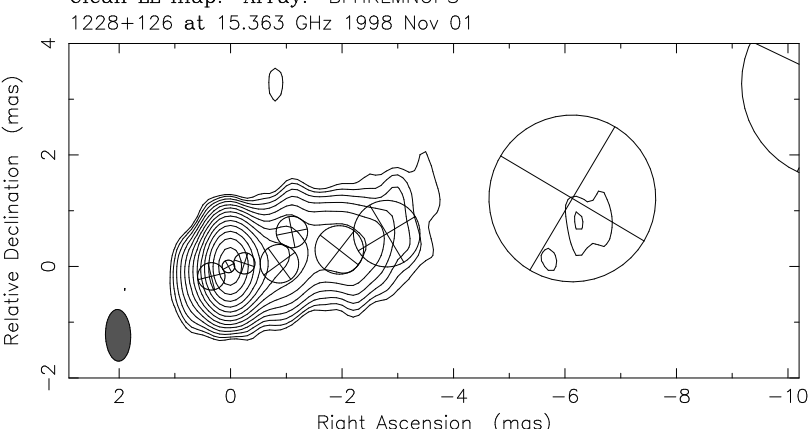

Map center: RA: 123049.426 , Dec: +12 2328.119 (2000.0)

Map peak: $0.882 \mathrm{Jy} /$ beam

Contours \%: $0.55 \quad 0.825 \quad 1.24 \quad 1.862 .784 .186 .26$

Contours \%: 9.414 .121 .131 .747 .671 .4

Fig. A.2. Same as Fig. A.1, but for epochs: Mar. 1997-Nov. 1998.

Fig. A.1. VLBA images superimposed by our model-fits for M 87 (epoch: Jul. 1995-Oct. 1995). 
S. Britzen et al.: A new view on the M 87 jet origin: Turbulent loading leading to large-scale episodic wiggling

Clean LL map. Array: BFHKLMNOPS

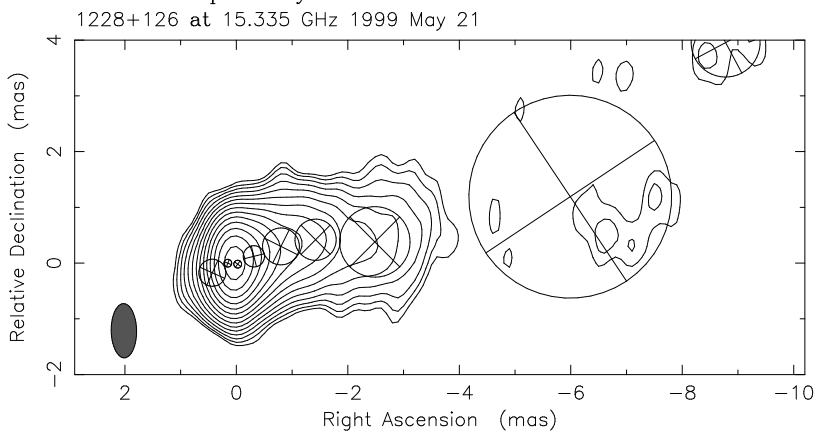

Map center: RA: 1230 49.423, Dec: +12 $2328.044(2000.0)$

Map peak: $0.784 \mathrm{Jy} /$ beam

Contours \%: $0.4 \quad 0.60 .91 .352 .033 .04 \quad 4.566 .83$

Contours \%: 10.315 .423 .134 .651 .977 .8

Beam FWHM: $0.974 \times 0.455$ (mas) at $0.891^{\circ}$

$3 \mathrm{C} 274$ at $15.365 \mathrm{GHz} 2000 \mathrm{Apr} 08$

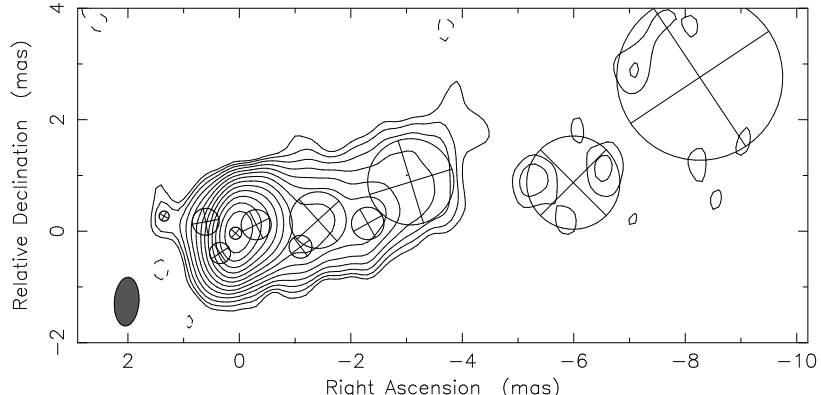

Map center: RA: 123049.423 , Dec: +122328.044 (2000.0)

Contours \%: 8.5412 .819 .228 .843 .264 .9

Clean I map Array: BFHKLMNOPS

$1228+126$ at $15.165 \mathrm{GHz} 2000$ Jun 27

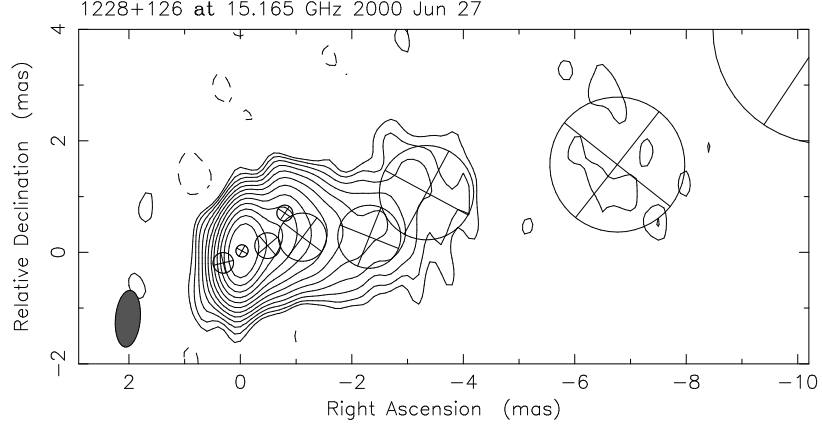

Map center: RA: 1230 49.423, Dec: +122328.044 (2000.0)

Map peak: $0.807 \mathrm{Jy} /$ beam

Contours \%: $-0.65 \quad 0.65 \quad 0.975 \quad 1.46 \quad 2.193 .294 .94$

Contours \%: 7.411 .116 .72537 .556 .2

Beam Film: $1.02 \times 0.446$ (mas) at 4.21010

Clean LL map. Array: BFHKLMOPS

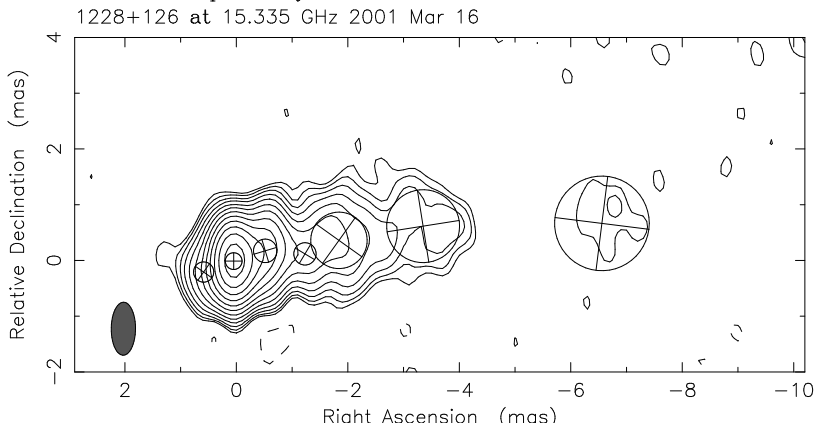

Map center: RA: 123049.423 , Dec: $+122328.044(2000.0)$

Map peak: $0.838 \mathrm{Jy} /$ beam

Contours \%: $-0.90 .91 .352 .023 .04 \quad 4.566 .83 \quad 10.3$

Contours \%: 15.423 .134 .651 .977 .8

Beam FWHM: $0.951 \times 0.436$ (mas) at $-0.476^{\circ}$

Fig. A.3. Same as Figs. A.1 and A.2, but for epochs: May 1999Mar. 2001.

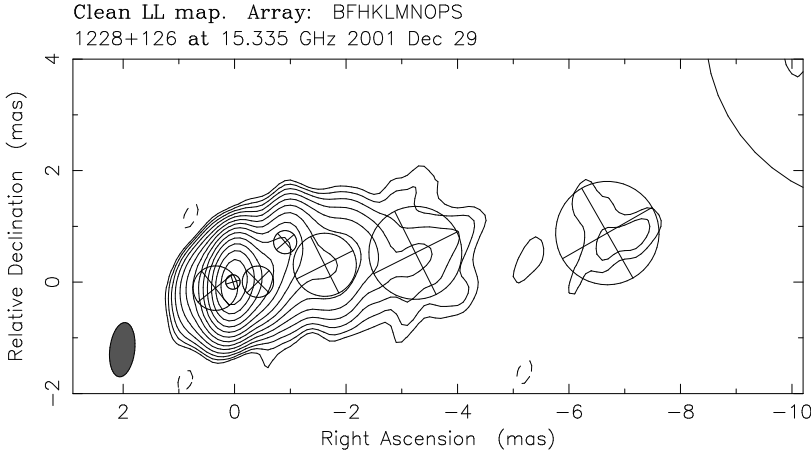

Map center: RA: 1230 49.423, Dec: +12 2328.044 (2000.0)

Map peak: $0.986 \mathrm{Jy} /$ beam

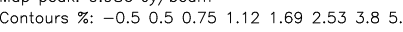

Contours \%: 8.5412 .819 .228 .843 .264 .9

Beam Fint. $0.979 \times 0.448$ (mas) at $-6.68^{\circ}$

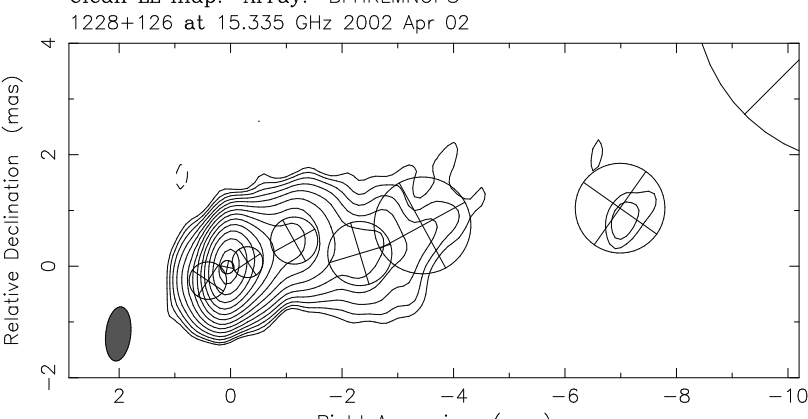

Map center: RA: 1230 49.423, Dec: +122328.044 (2000.0)

Map peak: 0.964

Contours \%: -0.60 .60 .91 .352 .033 .044 .566 .83$

Contours \%: 10.315 .423 .134 .651 .977 .8

Beam FWHM: $0.976 \times 0.45$ (mas) at $-6.14^{\circ}$

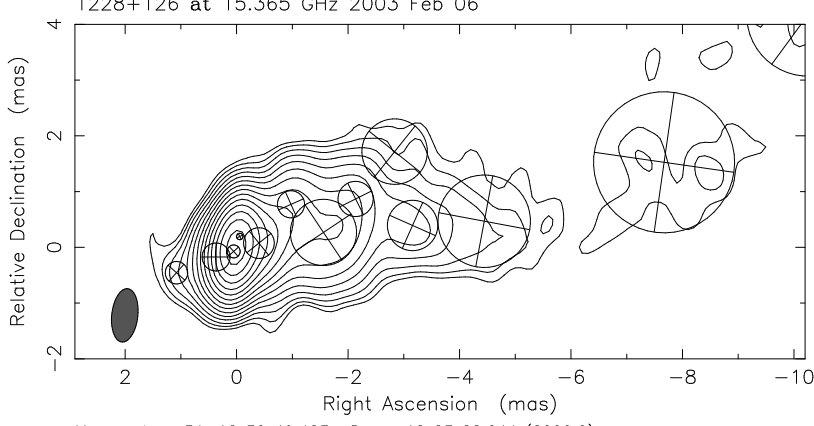

Map center: RA: 1230 49.423, Dec: +12 2328.044 (2000.0)

Map peak: $1.18 \mathrm{Jy} /$ beam

Contours \%: 0.40 .60 .91 .352 .033 .044 .566 .83

Contours \%: 10.315 .423 .134 .651 .977 .8

Com

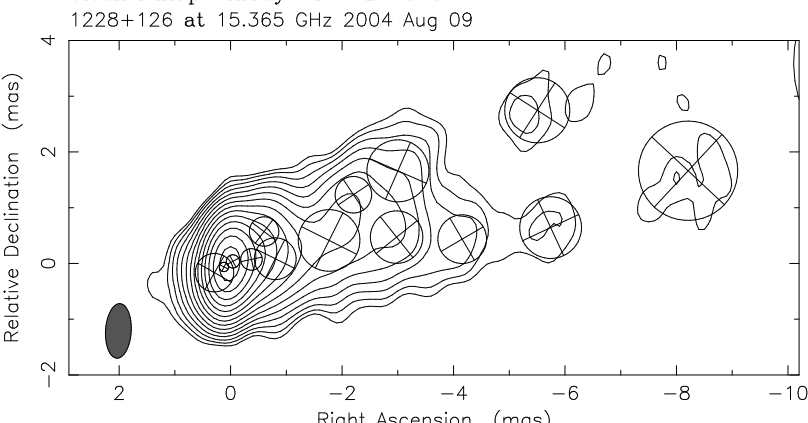

Map center: RA: 1230 49.423, Dec: $+122328.044(2000.0)$

Map peak: $0.898 \mathrm{Jy} /$ beam

Contours \%: 0.40 .60 .91 .352 .033 .044 .566 .83

Contours \%: 10.315 .423 .134 .651 .977 .8

Fig. A.4. Same as Figs. A.1-A.3, but for epochs: Dec. 2001-Aug. 2004 
Clean I map. Array: BFHKLMNOPS

$1228+126$ at $15.365 \mathrm{GHz} 2004 \mathrm{Dec} 03$

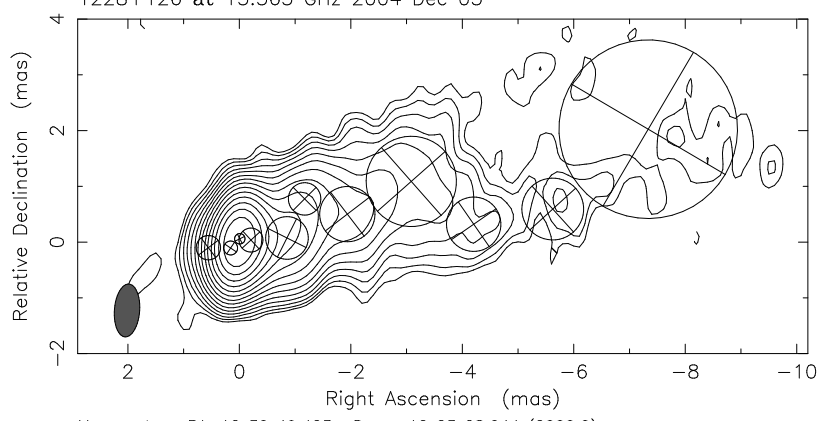

Map center: RA: 1230 49.423, Dec: $+122328.044(2000.0)$

Contours \%: $-0.3 \quad 0.3 \quad 0.45 \quad 0.675 \quad 1.01 \quad 1.52 \quad 2.28$

Contours \%: 3.425 .137 .6911 .517 .325 .938 .9

Contours \%: 58.4

0.454 (mas) at $-4.35^{\circ}$

Clean I map. Array: BFKLNOPH

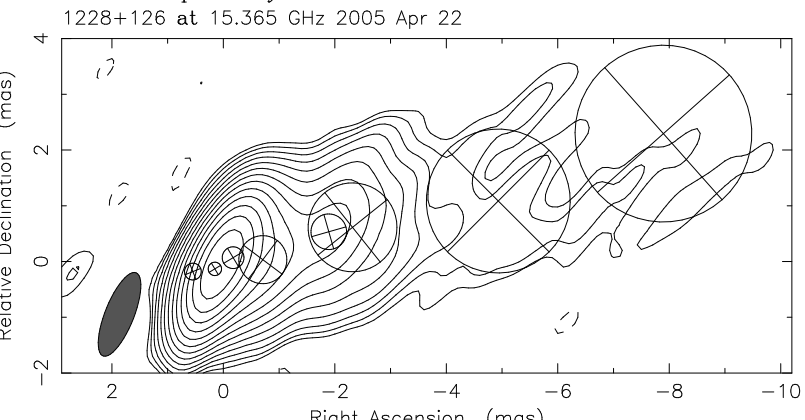

Right Ascension (mas)

Map center: RA: 1230 49.423, Dec: +122328.044 (2000.0)

Map peak: $1.01 \mathrm{Jy} /$ bean

0.50 .751121692533 .85 .7

Contours \%: 8.5412 .819 .228 .843 .264 .9

Beam FWHM: $1.61 \times 0.519$ (mas) at $-21.7^{\circ}$

$1228+126$ at $15.365 \mathrm{GHz} 2005 \mathrm{Nov} 07$

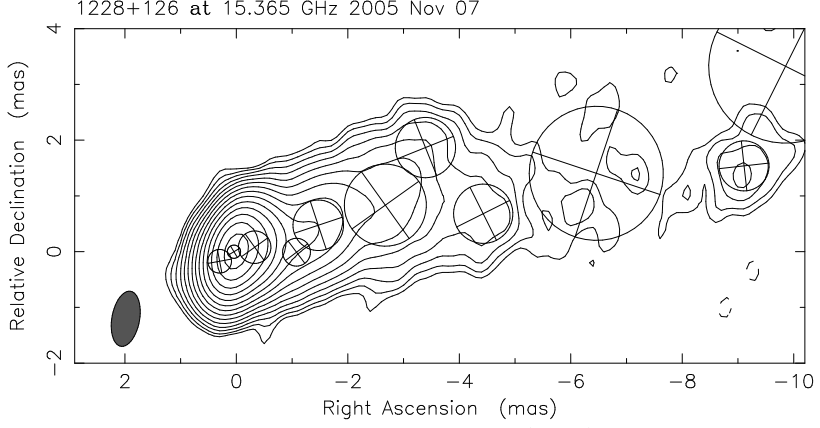

Map center: RA: 1230 49.423, Dec: $+122328.044(2000.0)$

Map peak: $0.871 \mathrm{Jy} /$ beam

Contours \%: $-0.40 .4 \quad 0.60 .91 .352 .033 .044 .56$

Contours \%: $6.83 \quad 10.315 .423 .134 .651 .977 .8$

Beam FWHM: 1 × 0.497 (mas) at $-10.2^{\circ}$

$1228+126$ at $15.365 \mathrm{GHz} 2006$ Jun 15

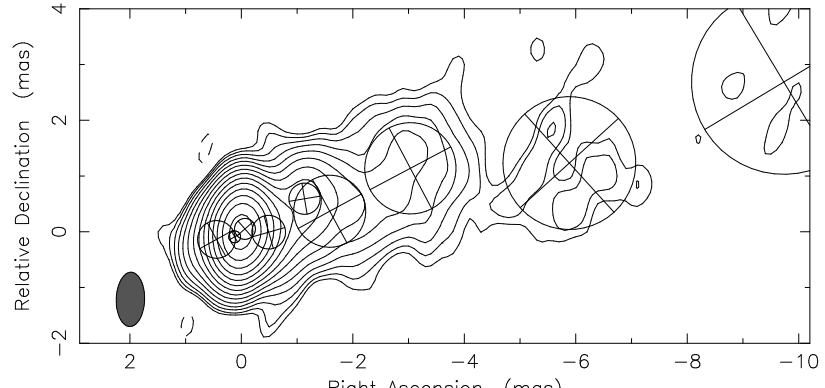

Right Ascension (mas)

Map center: RA: 123049.423 , Dec: $+122328.044(2000.0)$

Map peak: $1.09 \mathrm{Jy} /$ beam

Contours \%: -0.40 .40 .60 .91 .352 .033 .044 .56$

Contours \%: 6.8310 .315 .423 .134 .651 .977 .8

Fig. A.5. Same as Figs. A.1-A.4, but for epochs: Dec. 2004-Jun. 2006.
Clean I map Array: BFHKLMNOPS

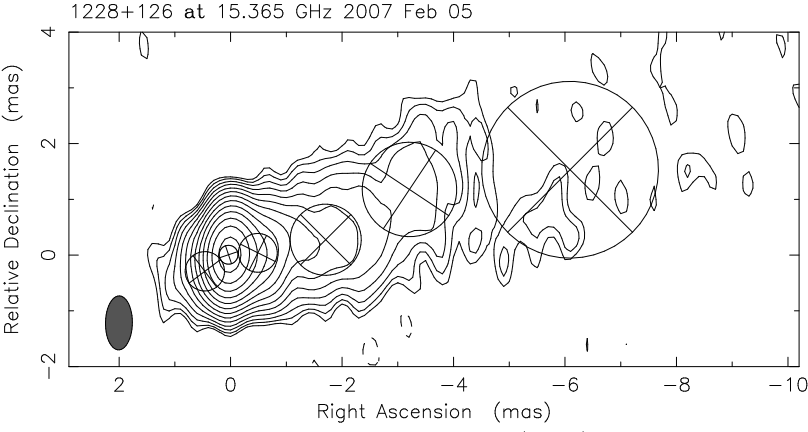

Map center: RA: 1230 49.423, Dec: +12 2328.044 (2000.0)

Map peak: $1.05 \mathrm{Jy} /$ beam

Contours \%: $-0.6 \quad 0.60 .91 .352 .033 .044 .566 .83$

Contours \%: 10.315 .423 .134 .651 .977 .8

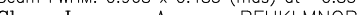

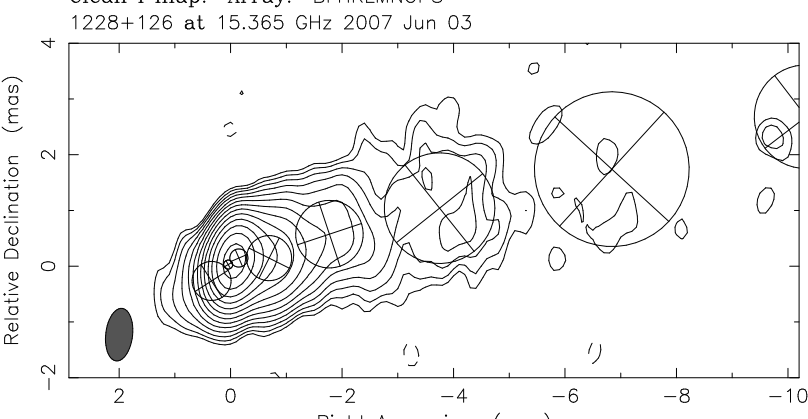

Map center: RA: 1230 49.423, Dec: +12 2328.044 (2000.0)

Map peak:

Contours \%: $-0.60 .6 \quad 0.91 .352 .023 .044 .566 .83$

Contours \%: 10.315 .423 .134 .651 .977 .8

Beam FWHM: $0.949 \times 0.482$ (mas) at $-6.66^{\circ}$

$1228+126$ at $15.365 \mathrm{GHz} 2008$

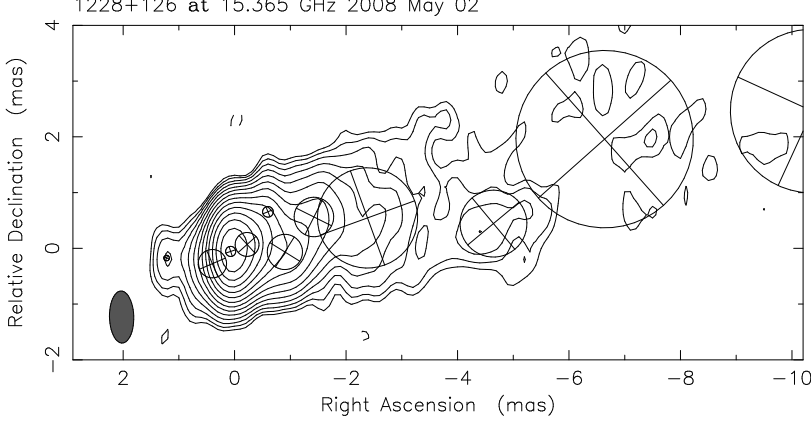

Map center: RA: 1230 49.423, Dec: +12 2328.044 (2000.0)

Map peak: 1.21 Jy/beam $1.00 .13 \quad 1.692 .53 \quad 3.85 .7$

Contours \%: 8.5412 .819 .228 .843 .264 .9

Cleam F I $0.035 \times 0.436$ (mas) at $1.39^{\circ}$

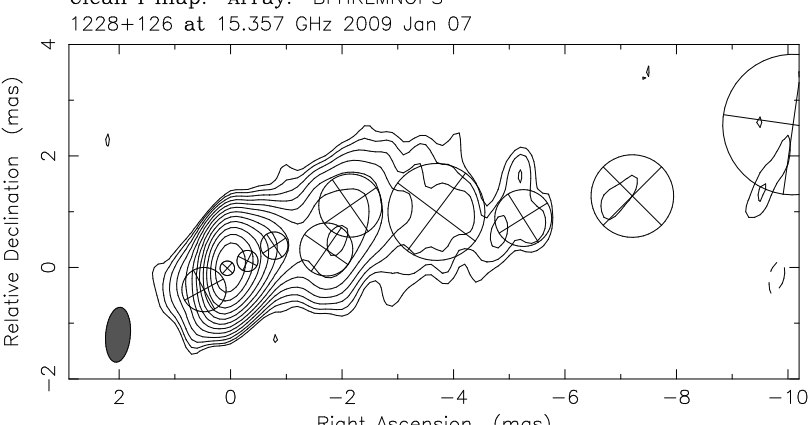

Map center: RA: 1230 49.423, Dec: +12 2328.044 (2000.0)

Map peak: $0.899 \mathrm{Jy} /$ bean

Contours \%: $-0.7 \quad 0.7 \quad 1.05 \quad 1.57 \quad 2.36 \quad 3.54 \quad 5.327 .97$

Contours \%: $12 \quad 17.926 .940 .460 .5$

FWHM: $0.988 \times 0.444$ (mas) at $-4.96^{\circ}$

Fig. A.6. Same as Figs. A.1-A.5, but for epochs: Feb. 2007-Jan. 2009. 
S. Britzen et al.: A new view on the M 87 jet origin: Turbulent loading leading to large-scale episodic wiggling

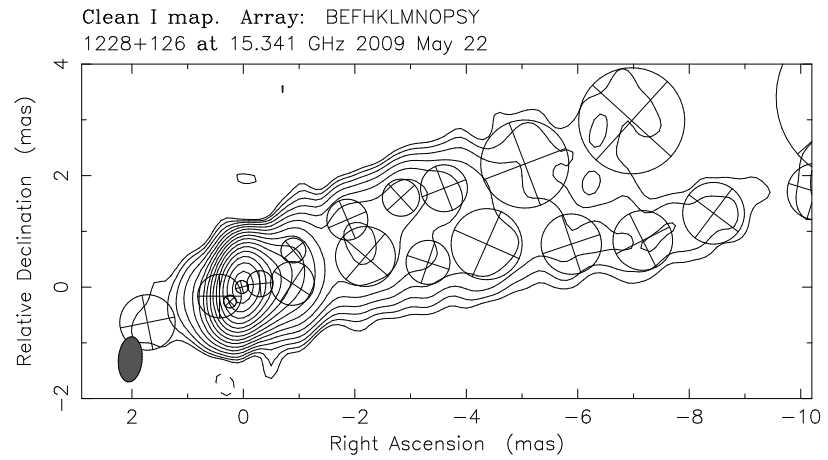

Map center: RA: 1230 49.423, Dec: $+122328.044(2000.0)$

Map peak: $1.03 \mathrm{Jy} /$ beam

Contours \%: $\begin{array}{llllllll}-0.25 & 0.25 & 0.375 & 0.562 & 0.844 & 1.27 & 1.9\end{array}$

Contours \%: 2.854 .27

Contours: $48.7733 .0 .427(\mathrm{mas})$ at

Clean I map. Array: BFHKLMNOPS

$1228+126$ at $15.357 \mathrm{GHz} 2009 \mathrm{Jul} 05$

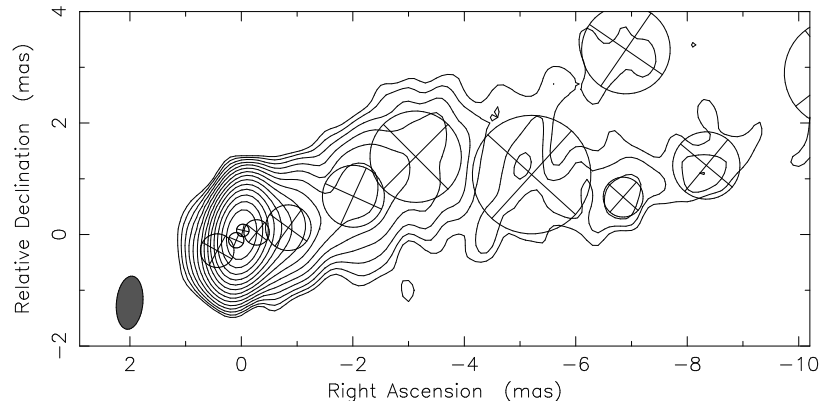

Map center: RA: 1230 49.423, Dec: +122328.044 (2000.0)

Map peak: $13 \mathrm{Jy} / \mathrm{beam}$

Contours \% 0.30 .450 .6751 .011 .5222 .283 .42

Contours \%. 5.137 .6911 .517 .325938958 .4

Beam FWHM: $0.957 \times 0.475$ (mas) at $-5.66^{\circ}$

Clean I map Array: BFKLMNOPS

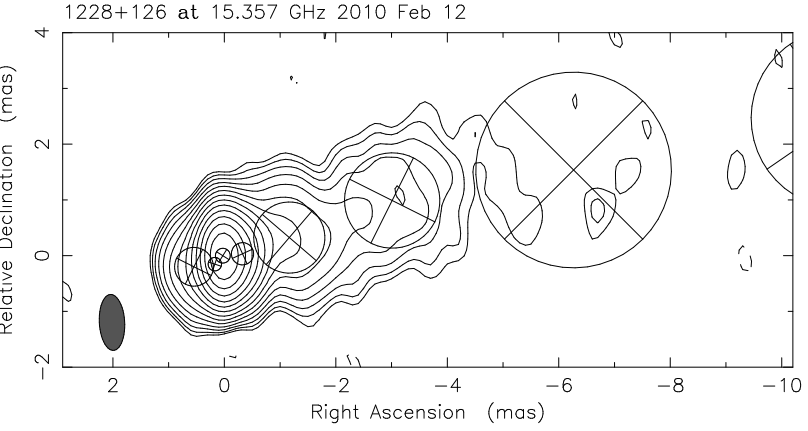

Map center: RA: 1230 49.423, Dec: $+122328.044(2000.0)$

Map peak: $1.01 \mathrm{Jy} /$ beam

Contours \%: $-0.5 \quad 0.5 \quad 0.751 .121 .692 .53 \quad 3.85 .7$

Contours \%: 8.5412 .819 .228 .843 .264 .9

Beam FWHM: $1.01 \times 0.455$ (mas) at $3.15^{\circ}$

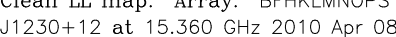

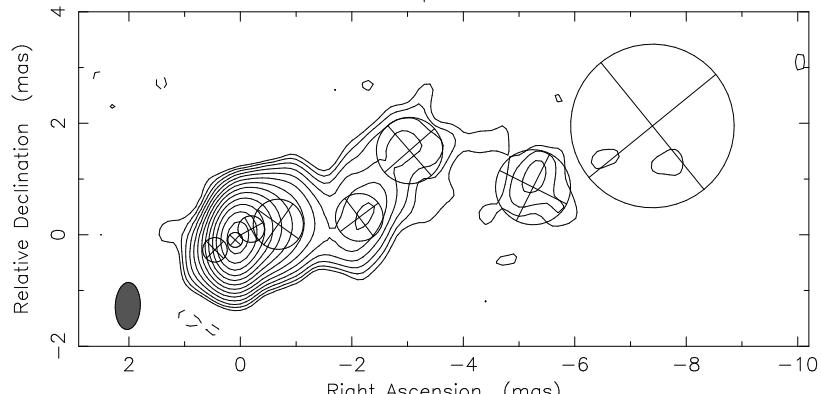

Right Ascension (mas)

Map center: RA: 123049.423 , Dec: +122328.044 (2000.0)

Map peak: $0.843 \mathrm{Jy} /$ beam

Contour -0.60 .60 .91352 .033 .044 .566 .83$

Contours \%: 10.315 .423 .134 .651 .977 .8

Fig. A.7. Same as Fig. A.1-A.6, but for epochs: May 2009-Apr. 2010.

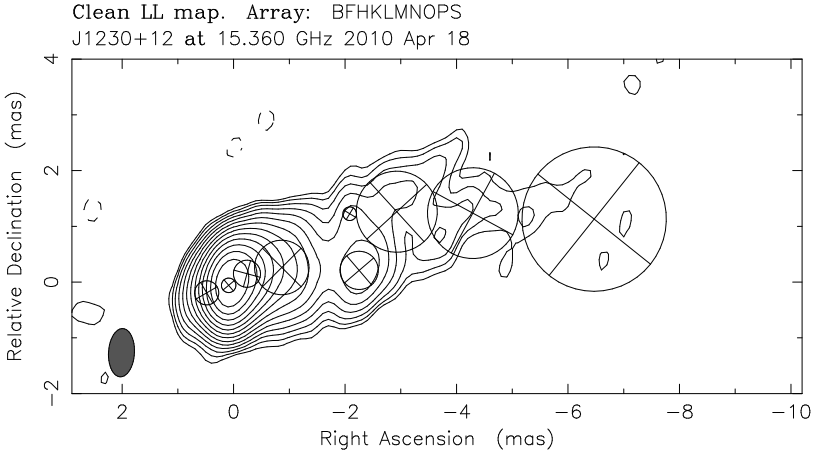

Map center: RA: 1230 49.423, Dec: +12 2328.044 (2000.0)

Map peak: $0.848 \mathrm{Jy} /$ beam

Contours \%: $-0.45 \quad 0.45 \quad 0.675 \quad 1.01 \quad 1.52 \quad 2.28 \quad 3.42$

Contours \%: 5.137 .6911 .517 .325 .938 .958 .4

Beam FMM. $0.868 \times 0.465$ (mas) at $-3.37^{\circ}$

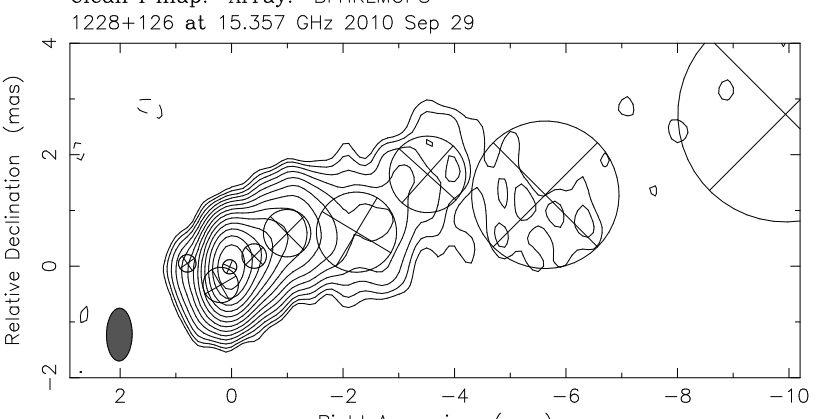

Map center: RA: 1230 49.423, Dec: $+122328.044(2000.0)$

Map peak: $0.931 \mathrm{Jy} /$ beam

Contours \%: -0.5050 .751 .121 .692 .533 .85 .7$

Contours \%: 8.5412 .819228 .843264 .9

Beam FWHM: $0.947 \times 0.461$ (mas) at $-0.712^{\circ}$

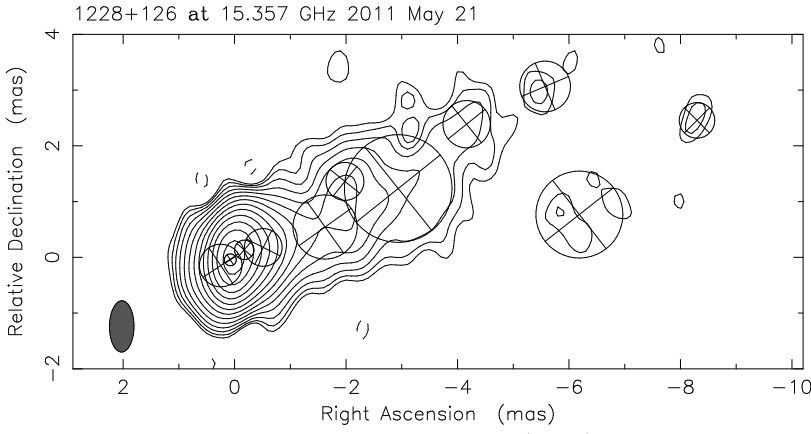

Map center: RA: 1230 49.423, Dec: +12 2328.044 (2000.0)

Map peak: $0.852 \mathrm{Jy} /$ beam

Contours \%: $-0.60 .6 \quad 0.91 .352 .03 \quad 3.044 .566 .83$

Contours \%: 10.315 .423 .134 .651 .977 .8

Fig. A.8. Same as Figs. A.1-A.7, but for epochs: Apr. 2010-May 2011. 